ARTICLE

\title{
TrkB-expressing paraventricular hypothalamic neurons suppress appetite through multiple neurocircuits
}

\author{
Juan Ji An¹, Clint E. Kinney¹, Ji-Wei Tan¹, Guey-Ying Liao', Eric J. Kremer² \& Baoji Xu (i) ${ }^{1 凶}$
}

The TrkB receptor is critical for the control of energy balance, as mutations in its gene (NTRK2) lead to hyperphagia and severe obesity. The main neural substrate mediating the appetite-suppressing activity of TrkB, however, remains unknown. Here, we demonstrate that selective Ntrk2 deletion within paraventricular hypothalamus (PVH) leads to severe hyperphagic obesity. Furthermore, chemogenetic activation or inhibition of TrkB-expressing PVH $\left(\mathrm{PVH}^{\mathrm{T} r \mathrm{~B}}\right)$ neurons suppresses or increases food intake, respectively. $\mathrm{PVH}^{\mathrm{TrkB}}$ neurons project to multiple brain regions, including ventromedial hypothalamus $(\mathrm{VMH})$ and lateral parabrachial nucleus (LPBN). We find that PVH TrkB neurons projecting to LPBN are distinct from those to $\mathrm{VMH}$, yet Ntrk2 deletion in PVH neurons projecting to either VMH or LPBN results in hyperphagia and obesity. Additionally, TrkB activation with BDNF increases firing of these PVH neurons. Therefore, TrkB signaling is a key regulator of a previously uncharacterized neuronal population within the PVH that impinges upon multiple circuits to govern appetite.

\footnotetext{
${ }^{1}$ Department of Neuroscience, The Scripps Research Institute Florida, Jupiter, FL 33458, USA. ${ }^{2}$ Institut de Génétique Moléculaire de Montpellier, University of Montpellier, CNRS, Montpellier, France. ${ }^{凶}$ email: bxu@scripps.edu
} 
T he regulation of food intake is essential for survival in all organisms, and its dysregulation not only affects survival but also causes health problems such as obesity. Several ligand-receptor pairs play a crucial role in the central regulation of energy balance. They include leptin and the leptin receptor, alpha-melanocyte-stimulating hormone (aMSH) and the melanocortin-4 receptor (MC4R), and brain-derived neurotrophic factor (BDNF) and the TrkB receptor. Defects in any of these three ligand-receptor pairs lead to severe obesity in humans ${ }^{1-5}$ and mice ${ }^{6-11}$. Furthermore, several common single nucleotide polymorphisms in the MC4R and BDNF genes have been found to be associated with increased body mass index in large-scale genome-wide association studies ${ }^{12-15}$. Remarkable progress has been made in elucidation of neural circuits that mediate the effects of leptin and $\alpha \mathrm{MSH}$ on energy balance ${ }^{16}$. By comparison, much less is known about the neural substrate through which the BDNF-TrkB pathway regulates appetite and energy expenditure.

BDNF is well known for its role in neuronal development and synaptic function ${ }^{17-19}$. It signals through the binding of two distinct classes of receptor proteins: the tropomyosin receptor kinase $B(T r k B)$ and the $p 75$ neurotrophin receptor $\left(p 75^{\mathrm{NTR}}\right)$. Mutations in the NTRK2 gene, which codes for TrkB, lead to severe obesity in mice and humans ${ }^{4,11}$. Conversely, no obesity phenotype has been observed in mice lacking the p75 ${ }^{\mathrm{NTR}}$ receptor ${ }^{20}$. In fact, mice lacking the $\mathrm{p} 75^{\mathrm{NTR}}$ receptor are protected from obesity induced by high-fat diet and remain lean due to increased energy expenditure ${ }^{21}$. Therefore, BDNF should signal through TrkB to regulate energy balance. The paraventricular hypothalamus (PVH) and the ventromedial hypothalamus (VMH) have been identified as two important brain areas that express BDNF to suppress food intake and promote energy expenditure ${ }^{22-24}$. However, it remains unclear in which population of hypothalamic neurons TrkB is required for the control of energy balance and how these TrkB-expressing neurons regulate food intake and/or energy expenditure. Our recent work found that Ntrk2 deletion in the dorsomedial hypothalamus (DMH) led to modest hyperphagia and obesity ${ }^{25}$, but to a much lesser extent in comparison with Ntrk2 hypomorphic mice in which Ntrk2 expression is reduced to a quarter of the normal amount throughout the body ${ }^{11}$, indicating that the main neural substrate underlying the action of TrkB signaling on energy balance remains to be identified.

Here we report that TrkB-expressing PVH $\left(\mathrm{PVH}^{\mathrm{TrkB}}\right)$ neurons are distinct from neurons that express BDNF, MC4R, glucagonlike peptide 1 receptor (GLP1R), or prodynorphin (PDYN) in the $\mathrm{PVH}$, the four subtypes of neurons that are known to suppress appetite 22,26-28. We find that chemogenetic inhibition of $\mathrm{PVH}^{\mathrm{TrkB}}$ neurons greatly increases food intake, whereas chemogenetic activation of these neurons drastically reduces food intake. Furthermore, we find that deletion of the Ntrk2 gene in the PVH leads to severe hyperphagic obesity. We then use a projection-specific gene deletion approach to reveal that $\mathrm{PVH}^{\mathrm{TrkB}}$ neurons project to the VMH and the lateral parabrachial nucleus (LPBN) to suppress food intake. These studies not only identify the main neural substrate by which TrkB signaling regulates energy balance, but also implicates the $\mathrm{PVH} \rightarrow \mathrm{VMH}$ neurocircuit in the control of appetite.

\section{Results}

Many TrkB neurons in the PVH are distinct from known ones. The PVH is a heterogeneous brain structure with many different cell types ${ }^{29,30}$. At least 11 types of PVH neurons can be molecularly defined by the expression of BDNF, corticotropinreleasing hormone $(\mathrm{CRH})$, growth hormone-releasing hormone
(GHRH), MC4R, oxytocin, PDYN, GLP1R, somatostatin, tyrosine hydroxylase, thyrotropin-releasing hormone (TRH), and vasopressin $22,26,27,31$. We previously found that approximately $5 \%$ $\mathrm{PVH}^{\mathrm{TrkB}}$ neurons expressed $\mathrm{BDNF}^{22}$. We sought to determine whether $\mathrm{PVH}^{\mathrm{TrkB}}$ neurons belong to other defined neuronal populations.

TrkB antibodies do not mark cell bodies well in brain sections, because TrkB is distributed to the membrane of both cell bodies and processes. We employed an Ntrk2 $2^{\mathrm{CrEER} /+}$ mouse $\operatorname{strain}^{32}$, in which the Cre-ERT2 sequence is inserted into the Ntrk2 locus immediately after the first coding ATG, for colocalization studies. We crossed Ntrk2 $2^{\mathrm{CreER} /+}$ mice to Rosa26 $6^{\mathrm{Ai} 9 /+}$ mice $^{33}$ to generate Ntrk2 $2^{C r e E R /+} ;$ Rosa26 $6^{\mathrm{Ai} 9 /+}$ mice, in which TrkB-expressing neurons should express tdTomato after tamoxifen treatment. Since the kinase-lacking truncated $\operatorname{TrkB}$ is expressed in astrocytes ${ }^{34}$, tdTomato should also label astrocytes. We found that $83 \%(1206 /$ 1449) of PVH cells containing Ntrk2 mRNA were positive for tdTomato mRNA, whereas 86\% (1206/1404) of PVH cells containing tdTomato mRNA were positive for Ntrk2 mRNA (Fig. 1a). Thus, tdTomato efficiently and faithfully marks TrkBexpressing cells in tamoxifen-treated $\mathrm{Ntrk} 2^{\mathrm{CreER} /+}$;Rosa26 $6^{\mathrm{Aig} /+}$ mice. We detected many tdTomato-labelled TrkB cells in the PVH (Fig. 1b), which are either neurons that are positive for the neuronal marker NeuN (Supplementary Fig. 1a) or astrocytes that are positive for glial fibrillary acidic protein (Supplementary Fig. 1b). We noticed that there was a high colocalization between TrkB and oxytocin in the PVH (18\% TrkB neurons express oxytocin and 51\% oxytocin neurons express TrkB; Fig. 1c) and between TrkB and GLP1R (14\% cells positive for Ntrk2 mRNA express Glp1r mRNA and 37\% cells positive for Glp1r mRNA express Ntrk2 mRNA; Fig. 1d). In addition, a small number of PVH $^{\text {TrkB }}$ neurons express TRH (2\% TrkB neurons express TRH and 12\% TRH neurons express TrkB; Supplementary Fig. 1c), PDYN (6\% TrkB neurons express PDYN and 17\% PDYN neurons express TrkB; Fig. 1e), somatostatin ( $2 \%$ TrkB neurons express somatostatin and 16\% somatostatin neurons express TrkB; Supplementary Fig. 1d) and GHRH (5\% TrkB neurons express GHRH and 30\% GHRH neurons express TrkB; Supplementary Fig. 1e). Note that higher percentages of $\mathrm{PVH}^{\mathrm{TrkB}}$ neurons are positive for these markers in the shown images (Fig. 1 and Supplementary Fig. 1), because the images were taken from the area where these markers are expressed. Very few $\mathrm{PVH}^{\mathrm{TrkB}}$ neurons express CRH (Supplementary Fig. 2a), tyrosine hydroxylase (Supplementary Fig. 2b), or vasopressin (Supplementary Fig. 2c). We also examined colocalization of TrkB with MC4R in the PVH using the BAC Mc4r-EGFP mouse strain ${ }^{35}$. We only detected low colocalization of TrkB with MC4R in the PVH of tamoxifen-treated $N$ trk2 ${ }^{C r e E R /+} ;$ Rosa $26^{A i 9 /+} ; M c 4 r-E G F P$ mice (3\% TrkB neurons express MC4R and 10\% MC4R neurons express TrkB; Fig. 1f). Because there are overlaps in expression between the aforementioned markers in the $\mathrm{PVH}^{22,28,36}$, the sum of TrkB neurons expressing these markers should be significantly lower than $51 \%$ of total PVH ${ }^{\mathrm{TrkB}}$ neurons (Supplementary Fig. 2d). These results indicate that the majority of $\mathrm{PVH}^{\mathrm{TrkB}}$ neurons are distinct from previously defined neuronal populations.

Deletion of the Ntrk2 gene with Sim1-Cre leads to obesity. We previously reported that Ntrk2 deletion in several hypothalamic areas using the Rgs9-Cre led to obesity ${ }^{37}$; however, the obesity phenotype was not as severe as what we observed in Ntrk2 hypomorphic mice that express $\operatorname{TrkB}$ at $\sim 25 \%$ of the normal amount throughout the body ${ }^{11,37}$. Given that Rgs9-Cre only deletes Ntrk2 in a small number of PVH neurons ${ }^{37}$ and that stereotaxic injection of recombinant BDNF into the PVH reduces 

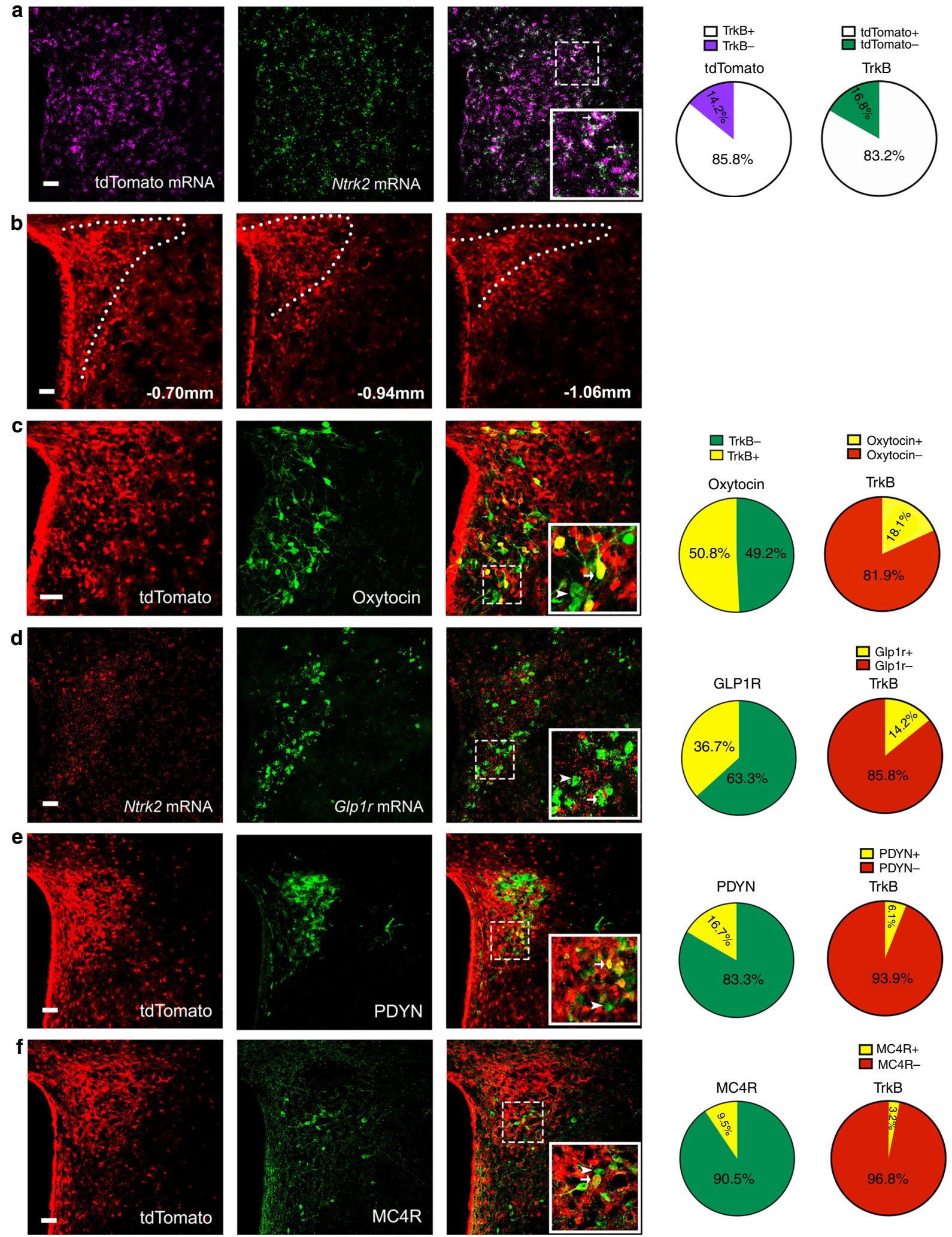

Fig. 1 Co-expression of TrkB with other neuronal markers in the PVH. TrkB-expressing cells are marked by tdTomato in tamoxifen-treated Ntrk2 CreER/t; Rosa26Ai9/+ mice. a Co-expression of tdTomato mRNA with Ntrk2 mRNA. b Distribution of TrkB-expressing cells in the PVH at three rostral-caudal positions. c-e Co-expression of TrkB with oxytocin, GLP1R, and PDYN. f Co-expression of tdTomato and EGFP in Ntrk2 $\mathrm{CreER/+}$; Rosa26 $6^{\mathrm{Aig} /+} ;$;Mc4r-EGFP mice. Arrows denote representative neurons expressing both TrkB and a PVH marker. Scale bars are $50 \mu \mathrm{m}$ long. Source data are provided as a Source Data file. 
food intake and increases energy expenditure ${ }^{38,39}$, this observation raises the possibility that TrkB expressed in the $\mathrm{PVH}(\mathrm{PVH}$ TrkB hereafter) plays an important role in the control of energy balance. We tested this possibility by deleting Ntrk2 in the PVH.

We tested whether Sim1-Cre ${ }^{40}$ is suitable for abolishment of Ntrk2 gene expression in the PVH using Sim1-Cre;Ntrk2 $2^{f B Z /+}$ mice. Ntrk $2^{f B Z}$ is a floxed allele and contains an inserted sequence encoding tau- $\beta$-galactosidase ${ }^{41,42}$. It expresses tau- $\beta$-galactosidase under the control of the endogenous Ntrk2 promoter after its floxed sequence is deleted by Cre recombinase. Therefore, tau- $\beta$ galactosidase expression in Sim1-Cre; Ntrk $2^{f B Z /+}$ mice indicates where the Sim1-Cre transgene could be used to abolish TrkB expression. We detected many cells expressing $\beta$-galactosidase in the PVH (Fig. 2a), suggesting that Sim1-Cre could be used to effectively abolish TrkB expression in the PVH. In addition to the PVH, Sim1-Cre could also abolish TrkB expression in some neurons of other brain regions, including the cerebral cortex, hippocampus, amygdala, thalamus, and $\mathrm{DMH}$ (Supplementary Fig. 3a-f).

We crossed Sim1-Cre mice to Ntrk $2^{\text {lox/lox }}$ mice, which express a normal amount of TrkB in the absence of $\mathrm{Cre}^{43}$, to produce Ntrk $2^{\text {lox/lox }}$ (control) and Sim1-Cre;Ntrk $2^{\text {lox/lox }}$ (mutant) mice. In situ hybridization confirmed that Ntrk2 expression was abolished in the PVH of Sim1-Cre;Ntrk2lox/lox mice (Fig. 2b). Male and female Sim1-Cre;Ntrk2lox/lox mice at 16 weeks of age were 45 and $49 \%$ heavier than sex-matched control mice, respectively (Fig. 2c, d). The Sim1-Cre;Ntrk2lox/lox mice also had a longer body (Fig. 2e) and larger adipose tissues relative to control mice (Supplementary Fig. 3g, h). These results indicate that Sim1-Cre; Ntrk2lox/lox mice develop obesity.

At 8 weeks of age, female Sim1-Cre;Ntrk2lox/lox mice daily consumed $24 \%$ more food than control mice (Fig. 2f). We employed indirect calorimetry to estimate energy expenditure in mice at 6 weeks of age when control and Sim1-Cre;Ntrk2lox/lox mice had comparable body weights (Fig. 2c). Control and Sim1Cre;Ntrk2 $2^{\text {lox } / l o x}$ mice had comparable oxygen consumption $\left(\mathrm{VO}_{2}\right)$ during the light period when mice are mostly at sleep (Fig. $2 \mathrm{~g}, \mathrm{~h}$ ), suggesting Sim1-Cre;Ntrk2lox/lox mice have a relatively normal basal metabolic rate. During the dark period, $\mathrm{VO}_{2}$ and locomotor activity in Sim1-Cre;Ntrk2lox/lox mice were reduced by 15 and $51 \%$, respectively, compared with control mice (Fig. $2 \mathrm{~g}-\mathrm{i}$ ). It is unlikely that the reduction in $\mathrm{VO}_{2}$ is secondary to the small, but statistically insignificant, excess weight gain in mutant mice, because the reduction did not occur during the light period (Fig. 2h). These results indicate that TrkB signaling in Sim1-Cre cells promote physical activity and energy expenditure. Collectively, our results indicate that Sim1-Cre;Ntrk2lox/lox mice develop obesity due to increased food intake and reduced energy expenditure.

Ntrk2 deletion in the adult PVH led to hyperphagic obesity. Sim1-Cre is expressed in other brain regions in addition to the $\mathrm{PVH}^{40}$ (Supplementary Fig. 3a-f), so the obesity phenotype in Sim1-Cre; Ntrk2lox/lox mice might result from Ntrk2 deletion in non-PVH neurons or cells. To validate a role for PVH TrkB in the regulation of energy balance, we stereotaxically injected neurontropic AAV2, either AAV2-CMV-Cre-GFP or AAV2-CMV-GFP, into the PVH of 8 -week-old female Ntrk $2^{\text {lox/lox }}$ mice (Fig. 3a). Injection of AAV2-CMV-Cre-GFP selectively abolished Ntrk2 expression in the PVH (Fig. 3a2, a3). Mice injected with AAV2CMV-Cre-GFP are divided into two groups based on the extent of AAV infection in the PVH: AAV2-CMV-Cre-GFP (missed) and AAV2-CMV-Cre-GFP (hit) (Supplementary Table 1). Mice in the AAV2-CMV-Cre-GFP (hit) group developed severe obesity quickly, compared to mice in the AAV2-CMV-GFP group and the
AAV2-CMV-Cre-GFP (Missed) group (Fig. 3b, c). The severity of obesity in AAV-Cre-injected mice had a good correlation with the extent of AAV infection in the PVH (Fig. 3d), but not along needle tracks or in other hypothalamic areas (Supplementary Table 1). These results show that PVH TrkB is critical for the control of energy balance.

Mice in the AAV2-CMV-Cre-GFP (hit) group displayed marked hyperphagia just two weeks after AAV injection and remained hyperphagic 10 weeks after injection (Fig. 3e). These mice had energy expenditure and respiratory exchange ratio comparable to control mice (Fig. 3f, h), whereas their ambulatory activity was trending lower 2 weeks after injection and was reduced 10 weeks after injection (Fig. $3 \mathrm{~g}$ ). These results indicate that PVH TrkB regulates energy balance mainly by suppressing appetite.

We noticed that the extent of AAV infection along a needle track is correlated with injection volume. We sought to further verify the role of PVH TrkB in the control of appetite by injecting female Ntrk2 $2^{\text {lox/lox }}$ mice with a smaller volume of AAV into the $\mathrm{PVH}$ to minimize infection in needle tracks and other hypothalamic nuclei. We detected very few AAV-transduced neurons in brain areas other than the PVH (mostly detected in Bregma -0.7 to $-0.94 \mathrm{~mm}$ ) in this batch of mice (Supplementary Fig. 4a1-a3). In the PVH, Ntrk2 gene deletion did not cause neuronal loss during the course of the experiment (Supplementary Fig. 4a4-a6). Mice injected with AAV2-CMV-Cre-GFP displayed the same phenotypes as we observed in the first batch of mice, i.e. severe hyperphagic obesity, increased linear growth and reduced ambulatory activity (Supplementary Fig. 4b-g). Interestingly, even unilateral Ntrk2 gene deletion was sufficient to cause significant obesity (Supplementary Fig. 4b), indicating that normal energy homeostasis needs full-strength TrkB signaling in PVH neurons that control appetite. Furthermore, Ntrk2 gene deletion in the PVH also led to obesity in male mice (Supplementary Fig. 4h). We did not detect any toxic effect on $\mathrm{PVH}$ neurons from AAV transduction, as bilateral injection of AAV2-CMV-Cre-GFP into the PVH of wild-type mice did not alter body weight (Supplementary Fig. 4i).

Although studies suggest that MC4R signaling interacts with BDNF-TrkB signaling in the context of body weight regulation $^{11,44}$, it is unlikely that Ntrk2 deletion in the PVH leads to obesity through the MC4R because the deletion did not reduce levels of Mc4r mRNA (Supplementary Fig. 4j, k). We also investigated whether PVH TrkB controls energy balance by regulating the function of oxytocin neurons, as many oxytocin neurons express TrkB (Fig. 1c) and oxytocin neurons may play a central role in the control of appetite ${ }^{45-49}$. We deleted the Ntrk2 gene in oxytocin neurons using the Oxt ${ }^{C r e}$ mouse strain $^{50}$ (Supplementary Fig. 5a) and found that the deletion did not affect body weight in mice of either sex (Supplementary Fig. 5b, c).

Collectively, these selective gene deletion experiments show that signaling through the TrkB receptor potently suppresses appetite through non-oxytocin-expressing PVH neurons. They also indicate that TrkB signaling in the PVH somewhat promotes locomotion without significantly altering energy expenditure.

PVH ${ }^{\text {TrkB }}$ neurons bidirectionally regulate food intake. As BDNF potently potentiates neuronal growth and synaptic function $^{19}$, Ntrk2 deletion would impair the function of PVH ${ }^{\text {TrkB }}$ neurons. The observation that Ntrk2 deletion in the PVH leads to hyperphagic obesity would implicate $\mathrm{PVH}^{\mathrm{TrkB}}$ neurons in the acute regulation of food intake. We tested this hypothesis by manipulating the activity of $\mathrm{PVH}^{\mathrm{TrkB}}$ neurons using designer receptors exclusively activated by designer drugs (DREADDs) 
a

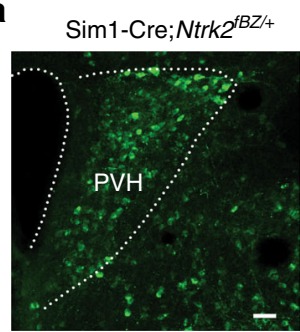

b

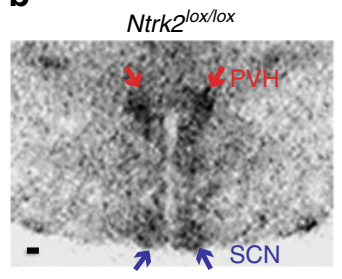

Sim1-Cre; Ntrk2 $2^{10 x / 10 x}$

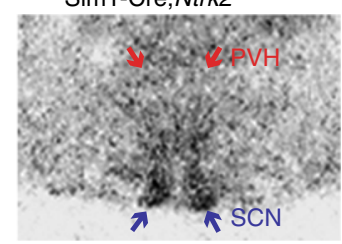

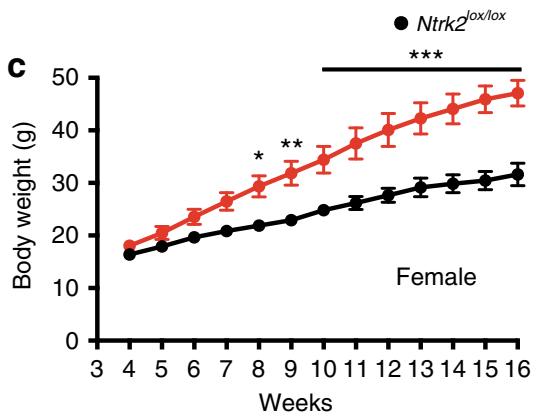

e

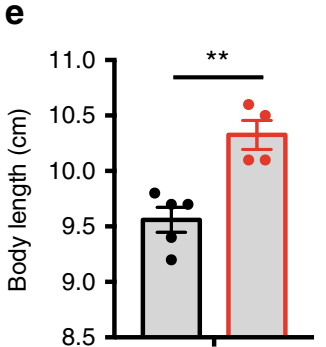

$\mathbf{f}$
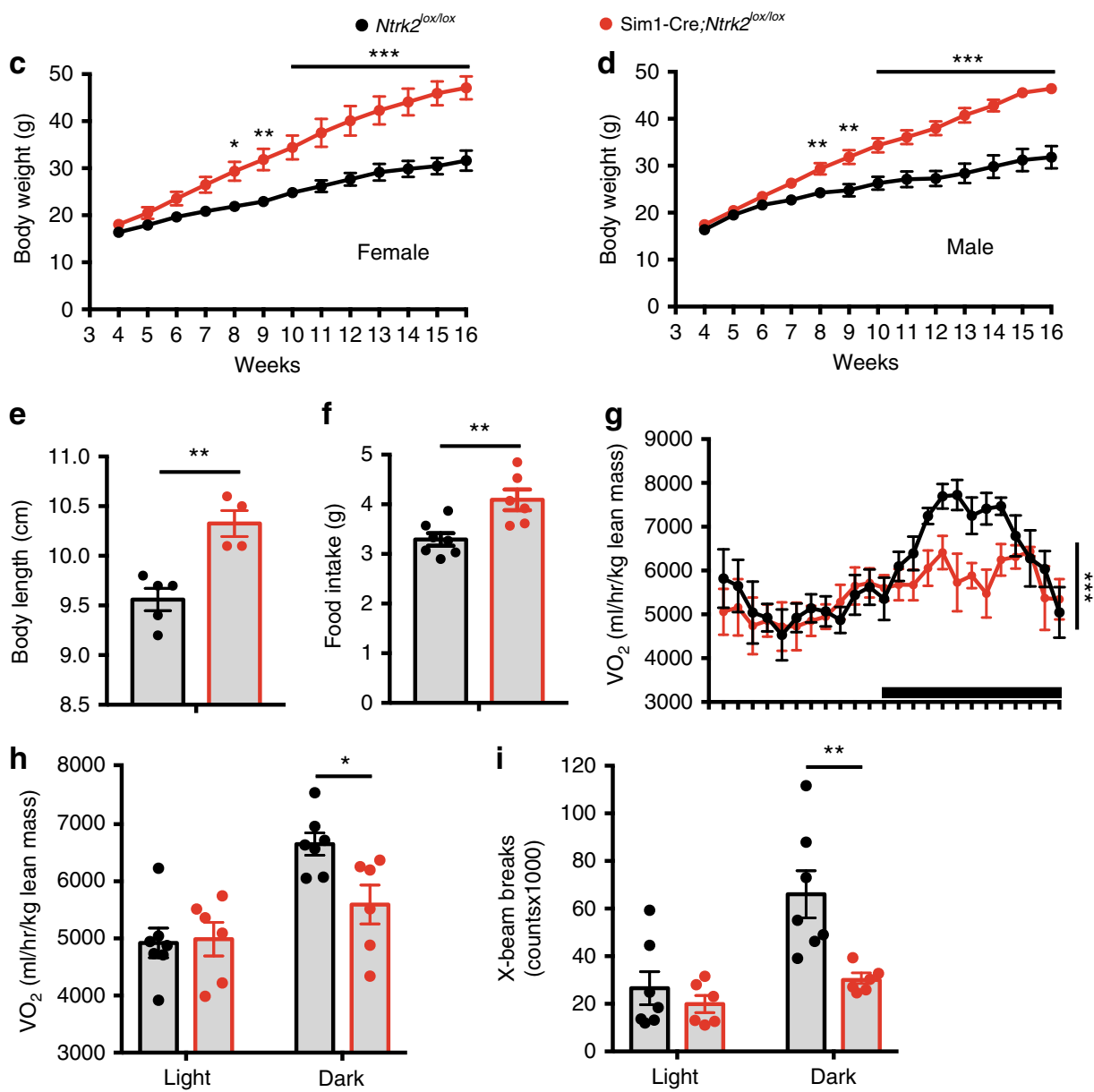

Fig. 2 Deletion of the Ntrk2 gene in Sim1-Cre cells led to obesity. a $\beta$-galactosidase immunohistochemistry of Sim1-Cre; Ntrk2fBZ/+ mice. Scale bar, $50 \mu m$. b In situ hybridization of brain sections from 4-month-old Ntrk2lox/lox mice (control) and Sim1-Cre;Ntrk2 lox/lox mice (mutant) reveals that Ntrk2 gene expression was abolished in the PVH of mutant mice. Arrows denote the PVH and the suprachiasmatic nucleus (SCN). Scale bar, $200 \mu \mathrm{m}$. c Body weight of female control and mutant mice. $n=5$ mice per genotype. Two-way ANOVA with post hoc Bonferroni multiple comparisons; $F_{(1,104)}=163.7, P<0.0001$ for genotype; ${ }^{\star} P<0.05,{ }^{\star \star} P<0.01$, and ${ }^{\star \star \star} P<0.001$. d Body weight of male control and mutant mice. $n=4$ controls and six mutants. Two-way ANOVA with post hoc Bonferroni multiple comparisons; $F_{(1,104)}=193.5, P<0.0001$ for genotype; ${ }^{\star \star} P<0.01$ and ${ }^{\star \star \star} P<0.001$. e Body length of 8 -week-old female control and mutant mice. $n=5$ controls and four mutants. Two-tailed unpaired $t$ test; ${ }^{\star \star} P=0.003$. $\mathbf{f}$ Daily food intake of 8 -week-old female control and mutant mice. $n=7$ controls and six mutants. Two-tailed unpaired $t$ test; ${ }^{\star \star} P=0.006$. $\mathbf{g}$ Distribution of oxygen consumption $\left(\mathrm{VO}_{2}\right)$ over a 24-h period in 6week-old female mice. The black bar above $x$ axis indicates the dark cycle. $n=7$ controls and six mutants. Two-way ANOVA for genotype; $F_{(1,264)}=14.54$, ${ }^{\star \star \star} P=0.0002$. $\mathbf{h} \vee \mathrm{O}_{2}$ of 6 -week-old female mice during the light and dark cycles. $n=7$ controls and six mutants. Two-tailed unpaired $t$ test; ${ }^{\star} P=0.017$. i Locomotor activity of 6-week-old female mice during the light and dark cycles. $n=7$ controls and six mutants. Two-tailed unpaired $t$ test; ${ }^{\star \star} P=0.007$. Error bars indicate SEM. Source data are provided as a Source Data file.

and their ligand clozapine $\mathrm{N}$-oxide $(\mathrm{CNO})^{51,52}$. We expressed stimulatory DREADD hM3D(Gq), inhibitory DREADD hM4D (Gi), or control mCherry in PVH TrkB neurons by injecting Credependent AAV2-hSyn-DIO-hM3D(Gq)-mCherry, AAV2-hSynDIO-hM4D(Gi)-mCherry, or AAV2-hSyn-DIO-mCherry into the PVH of Ntrk2 $2^{C r E R} /+$ mice. We subsequently induced the expression of the injected AAV vectors in $\mathrm{PVH}^{\mathrm{TrkB}}$ neurons by treating the mice with tamoxifen (Fig. $4 \mathrm{a}, \mathrm{b}$ ).
Administration of $\mathrm{CNO}$ did not alter food intake of Ntrk2 $2^{C r E R /}+$ mice expressing mCherry during either the light or dark cycle or after fasting (Fig. $4 \mathrm{c}-\mathrm{f}$ ), indicating that $\mathrm{CNO}$ does not have any detectable nonspecific effect on feeding. Administration of CNO greatly increased food intake of Ntrk2 $2^{C r e E R /+}$ mice expressing hM4D(Gi)-mCherry during the light cycle when mice do not normally eat much food, compared with vehicle administration (Fig. 4c). This result indicates that $\mathrm{PVH}^{\mathrm{TrkB}}$ 
a

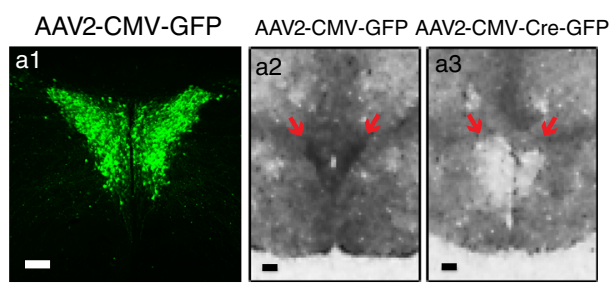

b

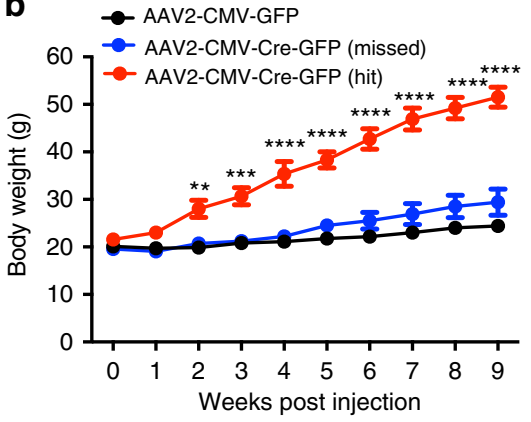

C

- AAV2-CMV-GFP

- AAV2-CMV-Cre-GFP (hit)

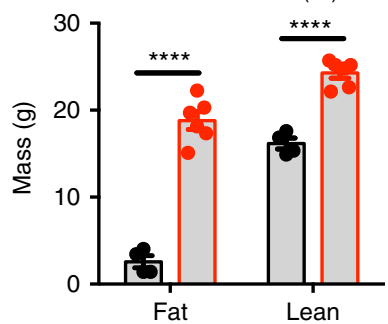

- AAV2-CMV-GFP AAV2-CMV-Cre-GFP (hit)

d

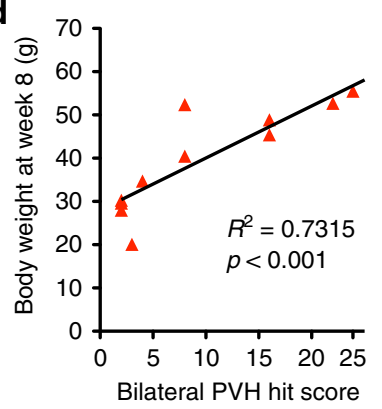

g

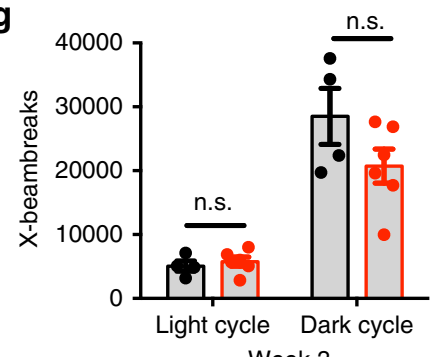

Week 3

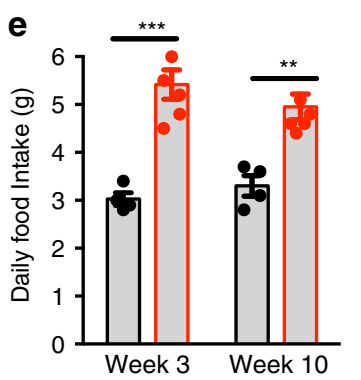

- AAV2-CMV-GFP

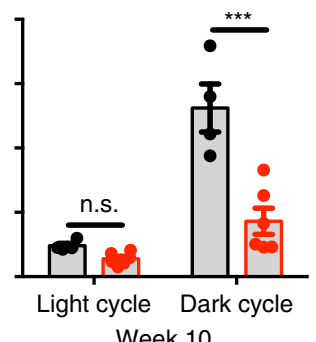

f

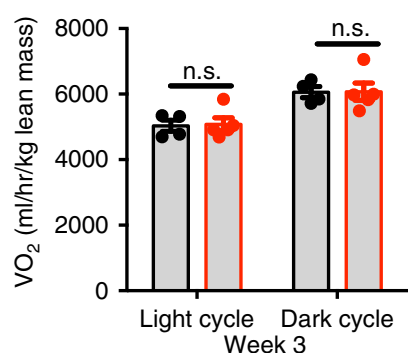

Week 3

- AAV2-CMV-Cre-GFP (hit)

h

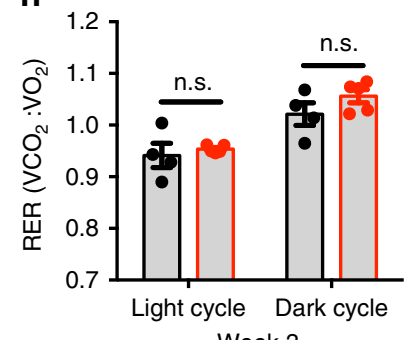

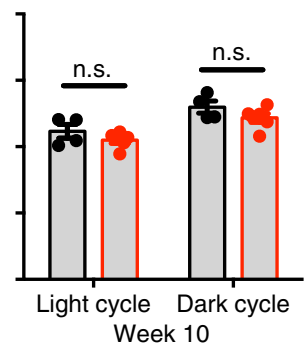

Week 10

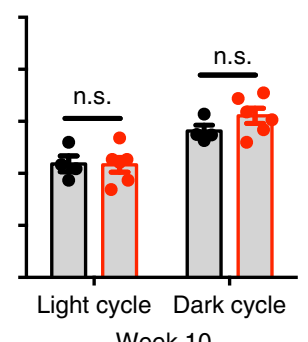

Week 10

Fig. 3 Deletion of the Ntrk2 gene in the PVH of adult female mice leads to hyperphagic obesity. a Bilateral injection of AAV2-CMV-GFP or AAV2-CMVCre-GFP (200 nl) into the PVH of Ntrk2lox/lox mice. GFP fluorescence (a1) and Ntrk2 mRNA in situ hybridization (a2, a3) are shown. Scale bars are $100 \mu \mathrm{m}$ (a1) and $200 \mu \mathrm{m}$ (a2, a3), respectively. b Body weight of Ntrk2lox/lox mice injected with either AAV2-CMV-GFP or AAV2-CMV-Cre-GFP into the PVH bilaterally. $n=4$ mice for AAV2-CMV-GFP, 5 mice for AAV2-CMV-Cre-GFP (missed), and 6 mice for AAV2-CMV-Cre-GFP (hit). Two-way ANOVA with post hoc Bonferroni multiple comparisons; $F_{(2,108)}=34.442, P<0.0001$ for viral injection; ${ }^{\star \star} P=0.0025$ and ${ }^{\star \star \star \star} P<0.0001$ for comparisons between AAV2-CMV-Cre-GFP (hit) and AAV2-CMV-GFP or AAV2-CMV-Cre-GFP (missed). c Body composition of Ntrk2lox/lox mice 9 weeks after AAV injection. $n=4$ mice for AAV2-CMV-GFP and 5 mice for AAV2-CMV-Cre-GFP (hit). Two-tailed unpaired $t$ test; $\star \star \star \star ~ P<0.0001$. d Correlation between the extent of AAV2-CMV-Cre-GFP transduction in PVH and body weight at 8 weeks after injection. Data are from both AAV2-CMV-Cre-GFP (hit) and AAV2-CMVCre-GFP (missed) groups. PVH hit score is a sum of the extents of AAV transduction in 6 divisions of the PVH (see Supplementary Table 1 for detail). e Daily food intake of Ntrk2 $2^{l o x} / l o x$ mice during week 3 and week 10 post injection. $n=4$ mice for AAV2-CMV-GFP and five mice for AAV2-CMV-Cre-GFP (hit). Two-tailed unpaired $t$ test; ${ }^{\star \star} P=0.0058$ and ${ }^{\star \star \star} P=0.0008 . \mathrm{f}_{2}$ consumption of Ntrk2lox/lox mice during week 3 and week 10 post injection. Twotailed unpaired $t$ test; n.s. not significant. $\mathbf{g}$ Locomotor activity of Ntrk $2^{\text {lox } / l o x}$ mice during week 3 and week 10 post injection. Two-tailed unpaired $t$ test; n.s. not significant and ${ }^{\star \star} P<0.002$. h RER of Ntrk2 ${ }^{10 x / l o x}$ mice during week 3 and week 10 post injection. Two-tailed unpaired $t$ test; n.s. not significant. Animal numbers for $\mathbf{f}-\mathbf{h}$ are 4 mice for AAV2-CMV-GFP and six mice for AAV2-CMV-Cre-GFP (hit). Error bars indicate SEM. Source data are provided as a Source Data file.

neurons are active to maintain physiological satiation during the light cycle. Interestingly, inhibition of $\mathrm{PVH}^{\mathrm{TrkB}}$ neurons also increased food intake during the dark cycle when mice are physiologically hungry and ingest the vast majority of their daily energy intake (Fig. 4d), suggesting that the anorexigenic $\mathrm{PVH}^{\mathrm{TrkB}}$ neurons are not fully silenced when mice are physiologically hungry. Conversely, $\mathrm{CNO}$ administration drastically reduced food intake of $\mathrm{Ntrk2} 2^{\mathrm{CreER} /+}$ mice expressing hM3D(Gq)mCherry during the dark cycle or after overnight fasting, compared with vehicle administration (Fig. 4e, f). The treatment induced Fos expression almost exclusively in hM3D(Gq)mCherry-expressing $\mathrm{PVH}^{\mathrm{TrkB}}$ neurons (Supplementary Fig. 6).
These results indicate that activation of $\mathrm{PVH}^{\mathrm{TrkB}}$ neurons is sufficient to suppress appetite. Taken together, these chemogenetic experiments indicate that $\mathrm{PVH}^{\mathrm{TrkB}}$ neurons are partially silenced to allow for feeding when mice are hungry, while being active to maintain physiological satiation during the light cycle, and thus $\mathrm{PVH}^{\mathrm{TrkB}}$ neurons play a critical role in the regulation of appetite by promoting satiety.

PVH $^{\text {TrkB }}$ neurons project to multiple brain areas. We sought to identify the downstream sites through which $\mathrm{PVH}^{\mathrm{TrkB}}$ neurons regulate appetite. To map the projections of $\mathrm{PVH}^{\mathrm{TrkB}}$ neurons in the brain, we injected Cre-dependent AAV2-CAG-FLEX-tdTomato 
a

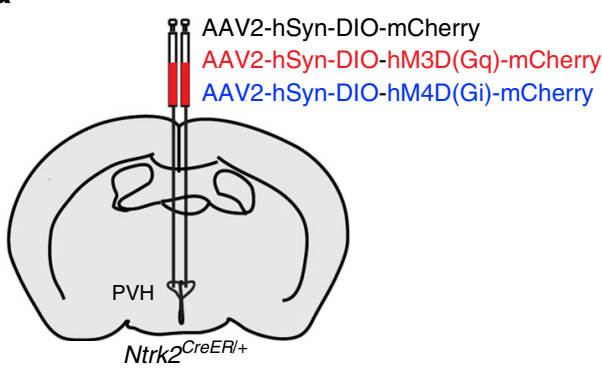

b

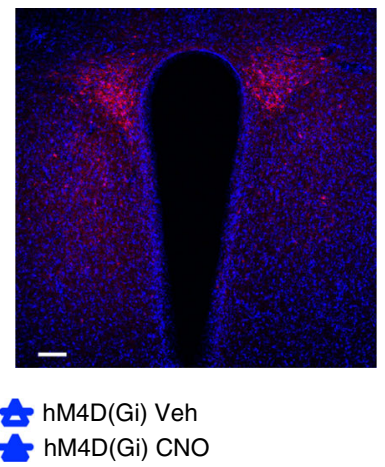

C

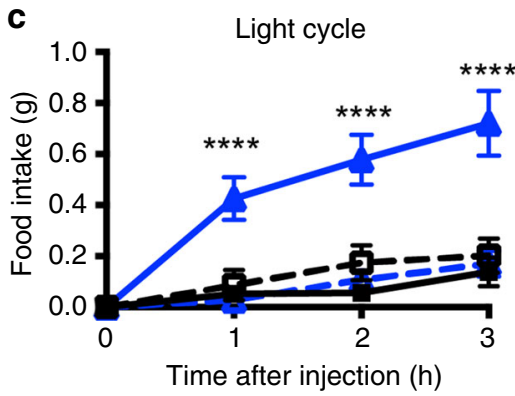

$\Xi$ mCherry Veh

\pm mCherry CNO

e

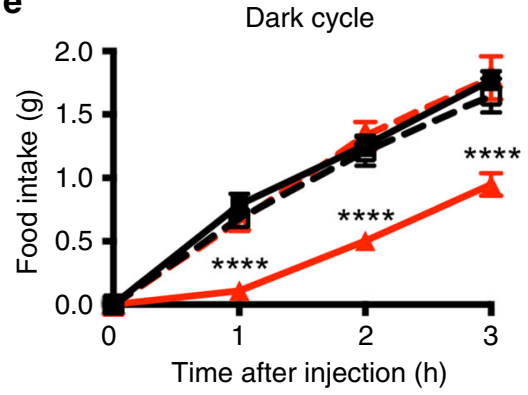

d

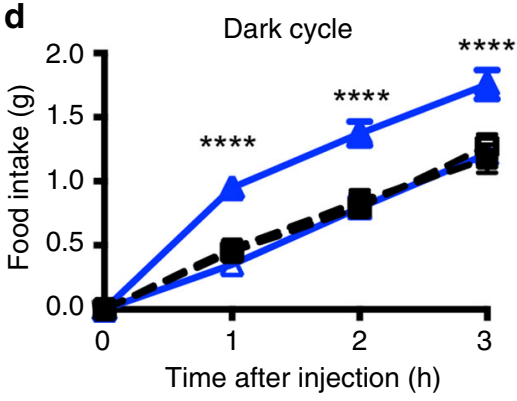

A hM3D (Gq) Veh $\pm \mathrm{hM} 3 \mathrm{D}(\mathrm{Gq}) \mathrm{CNO}$

$\mathbf{f}$

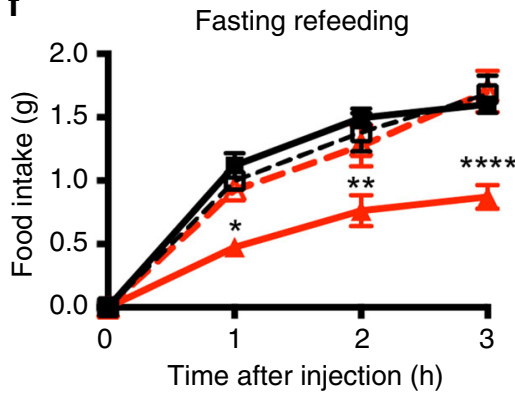

Fig. 4 Chemogenetic modification of PVH TrkB neuronal activity alters food intake. a A diagram showing bilateral injection of AAV2-hSyn-DIO-mCherry, AAV2-hSyn-DIO-hM3D(Gq)-mCherry, or AAV2-hSyn-DIO-hM4D(Gi)-mCherry into the PVH of Ntrk2CreER/+ mice. b A confocal imaging showing mCherry expression of injected AAV in the PVH. Brain sections were counter-stained with DAPI. The scale bar represents $100 \mu \mathrm{m}$. $\mathbf{c}$ Inhibition of PVH ${ }^{\text {TrkB }}$ neurons stimulated food intake during the light cycle. $n=5$ and six mice for AAV2-hSyn-DIO-mCherry (mCherry) and AAV2-hSyn-DIO-hM4D(Gi)mCherry $[\mathrm{hM} 4 \mathrm{D}(\mathrm{Gi})]$ groups, respectively. Two-way ANOVA with post hoc Tukey's multiple comparisons; $F_{(3,72)}=35.23, P<0.0001$ for virus and treatment; ${ }^{\star \star \star \star} p<0.0001$ when compared to the hM4D(Gi)-vehicle (Veh) group. d Inhibition of $\mathrm{PVH}^{\text {TrkB }}$ neurons stimulated food intake during the dark cycle. $n=6$ and six mice for mCherry and $\mathrm{hM} 4 \mathrm{D}(\mathrm{Gi})$ groups, respectively. Two-way ANOVA with post hoc Tukey's multiple comparisons; $F_{(3,76)}=31.49$, $P<0.0001$ for virus and treatment; ${ }^{\star \star \star \star} p<0.0001$ when compared to the $h M 4 D(G i)-V e h$ group. e Activation of $P V H^{T r k B}$ neurons suppressed food intake during the dark cycle. $n=6$ and seven mice for AAV2-hSyn-DIO-mCherry (mCherry) and AAV2-hSyn-DIO-hM3D(Gq)-mCherry [hM3D(Gq)] groups, respectively. Two-way ANOVA with post hoc Bonferroni's multiple comparisons; $F_{(3,22)}=17.27, P<0.0001$ for virus and treatment; $\star \star \star \star ~ P<0.0001$ when compared to the $h M 3 D(G q)-V e h$ group. $f$ Activation of PVH ${ }^{T r k B}$ neurons suppressed fasting-induced appetite. $n=6$ and seven mice for mCherry and $\mathrm{hM} 3 \mathrm{D}(\mathrm{Gq})$ groups, respectively. Two-way ANOVA with post hoc Bonferroni's multiple comparisons; $F_{(3,22)}=10.47, P<0.0001$ for virus and treatment; ${ }^{\star} P<0.05,{ }^{\star \star} P<0.01$, and ${ }^{\star \star \star \star} P<0.0001$ when compared to the $\mathrm{hM} 3 \mathrm{D}(\mathrm{Gq})$-Veh group. Error bars indicate SEM. Source data are provided as a Source Data file.

into the PVH of adult $\mathrm{Ntrk2} 2^{\mathrm{CrEER} /+}$ mice (Fig. 5a1). After tamoxifen treatment, tdTomato nicely labeled $\mathrm{PVH}^{\mathrm{T}}{ }^{\mathrm{T} B}$ neurons across the rostral-caudal axis (Fig. 5a2-4). $\mathrm{PVH}^{\text {TrkB }}$ neurons project to four main brain sites: the VMH (Fig. 5a5), the median eminence (ME; Fig. 5a5), the lateral parabrachial nucleus (LPBN; Fig. 5a6), and the nucleus tractus solitaris (NTS; Fig. 5a8). Lesser projections were detected in the medial parabrachial nucleus (MPBN; Fig. 5a6), the locus coeruleus (LC; Fig. 5a7), the dorsal motor nucleus of the vagus (DMV; Fig. 5a8), and the ventrolateral periaqueductal gray (vlPAG; Supplementary Fig. 7a). In the LPBN, axons of PVH TrkB neurons mainly terminate at the central compartment, which is next to the CGRP (calcitonin gene-related peptide)-marked external compartment $^{53}$ (Fig. 5b).

The projection to the ME is expected, as TrkB is expressed in a fraction of PVH neurons expressing GHRH (Supplementary Fig. 1e) and oxytocin (Fig. 1c), which project to the ME and the posterior pituitary via the ME, respectively, to release hormones. To validate other putative projection fields, we injected retrograde tracers, Alexa Fluor 488-conjugated cholera toxin subunit B (CTB488) or green retrobead (GRB), into each of these fields in 
a

a1
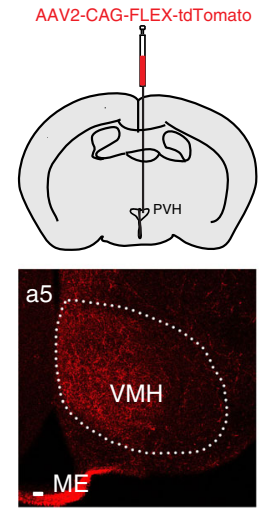

b

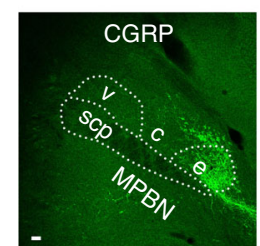

CTB488 injection
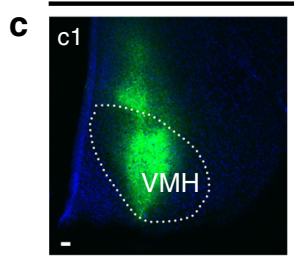
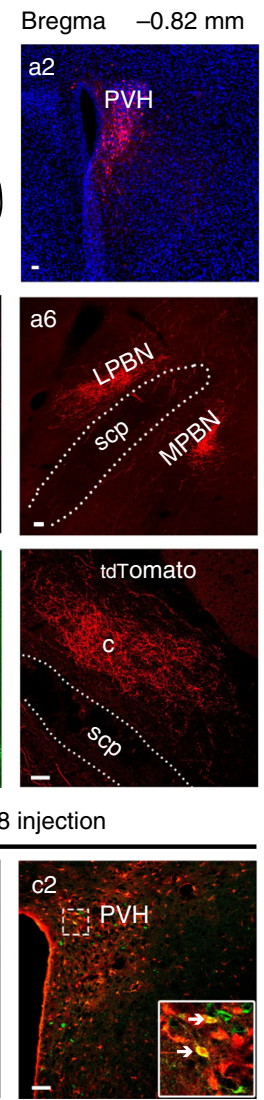
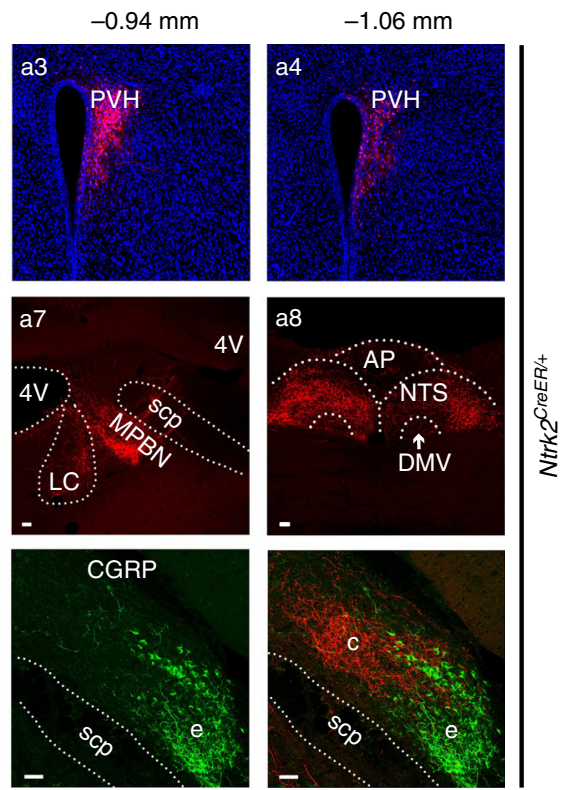

GRB injection

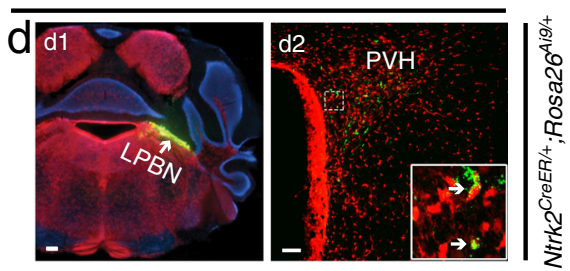

Fig. 5 Projections of PVHTrkB neurons. a AAV2-CAG-FLEX-tdTomato was unilaterally injected into the PVH of Ntrk2CreER/+ mice (a1). After the mice were treated with tamoxifen, tdTomato-labeled PVH TrkB neurons were present throughout the rostral-caudal axis (a2-a4). Axonal terminals of these neurons were found in the VMH, LPBN, MPBN, LC, NTS and DMV (a5-a8). b The LPBN includes the external (e), central (c), and ventral (v) compartments. Axonal terminals of $\mathrm{PVH}{ }^{\mathrm{TrkB}}$ neurons are mainly in the central compartment, which is next to the CGRP-marked external compartment. c, d Retrograde tracers, CTB488 (250 nl) or GRB (200 nl), were unilaterally injected into the VMH (c) or LPBN (d) of tamoxifen-treated Ntrk2CreER/+;Rosa26Ai9/+ mice and labeled some tdTomato-expressing PVHTrkB neurons. AP area postrema; DMV dorsal motor nucleus of the vagus; ME median eminence; LC locus coeruleus; LPBN lateral parabrachial nucleus; MPBN medial parabrachial nucleus; NTS nucleus tractus solitaris; PVH paraventricular hypothalamus; scp superior cerebellar peduncle; VMH ventromedial hypothalamus. Scale bars represent $200 \mu \mathrm{m}$ in d1 and $50 \mu \mathrm{m}$ in other panels. Source data are provided as a Source Data file.

tamoxifen-treated Ntrk2 $2^{C r E R /+} ;$ Rosa26 $6^{A i 9 /+}$ mice. In a true projection field, retrograde tracers would be taken up at axonal terminals and retrogradely transported to the cell bodies of $\mathrm{PVH}^{\mathrm{TrkB}}$ neurons. Indeed, we found that retrograde tracers injected into the VMH (Fig. 5c), LPBN (Fig. 5d), vlPAG, LC, and NTS (Supplementary Fig. 7) labelled some TrkB-expressing neurons in the $\mathrm{PVH}$. These results show that $\mathrm{PVH}^{\mathrm{T}} \mathrm{rB}$ neurons project to the central compartment of the LPBN (cLPBN), VMH, NTS, MPBN, DMV, vlPAG, ME, and pituitary.

Projection-specific gene deletion. In order to determine at which projection PVH TrkB is necessary for the regulation of appetite, we designed a two-virus system for projection-specific gene deletion. In this strategy, one viral vector is a retrograde virus [canine adenovirus $2(\mathrm{CAV} 2)^{54}$ or retrograde AAV (AAVretro $)^{55}$ ] expressing yeast flippase (FLP), whereas the other viral vector is AAV2-Efla-fDIO-mCherry-P2A-Cre, in which expression of mCherry-P2A-Cre is dependent on FLP-mediated recombination at two sets of Frt sequences (Fig. 6a). If we stereotaxically inject FLP-expressing retrograde virus into the LPBN and AAV2-Ef1afDIO-mCherry-P2A-Cre into the $\mathrm{PVH}$, the retrograde virus would be taken up at axonal terminals and retrogradely transported to the cell bodies of neurons that project to the LPBN. In the PVH, FLP expressed in neurons that project to the LPBN would mediate recombination between the two sets of Frt sites in AAV2-Ef1a-fDIO-mCherry-P2A-Cre and invert the orientation of the mCherry-P2A-Cre sequence, leading to the expression of mCherry and Cre (Fig. 6a). If the two viral vectors are injected into Ntrk2 $2^{l o x / l o x}$ mice, expressed Cre would delete the Ntrk2 gene in PVH neurons that project to the LPBN.

We conducted a proof-of-principle experiment to test this projection-specific gene deletion approach in the well-defined substantia nigra $\rightarrow$ striatum projection. We injected CAV2-FLPo, which expresses optimized FLP, into the striatum and AAV2Ef1a-fDIO-mCherry-P2A-Cre into the substantia nigra of $B d n f^{k l o x /+}$ mice (Fig. 6b1). Bdnfklox/+ mice contains a uniquely floxed $B d n f$ allele that starts to express $\beta$-galactosidase in BDNFexpressing cells when Cre recombines the allele ${ }^{56}$. We detected many mCherry-expressing neurons in the substantia nigra (Fig. 6b2), indicating that CAV2-FLPo was retrogradely transported to the substantia nigra from the striatum and induced mCherry expression from AAV2-Ef1a-fDIO-mCherry-P2A-Cre. Because mCherry and Cre are in the same cistron, mCherrypositive neurons should also express Cre, which would mediate recombination of the $B d n f^{k l o x}$ allele. Indeed, we found that some mCherry-positive neurons expressed $\beta$-galactosidase in the substantia nigra (Fig. 6b2-4), where $\sim 50 \%$ dopaminergic neurons express $\mathrm{BDNF}^{57}$. As expected, we detected expression of neither mCherry nor $\beta$-galactosidase in the substantia nigra 
a

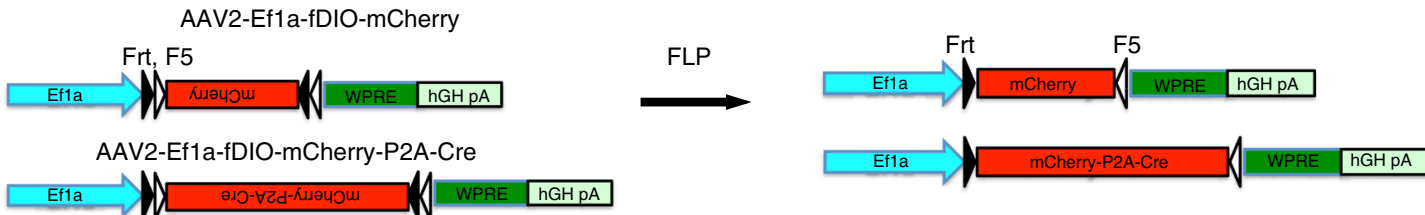

b
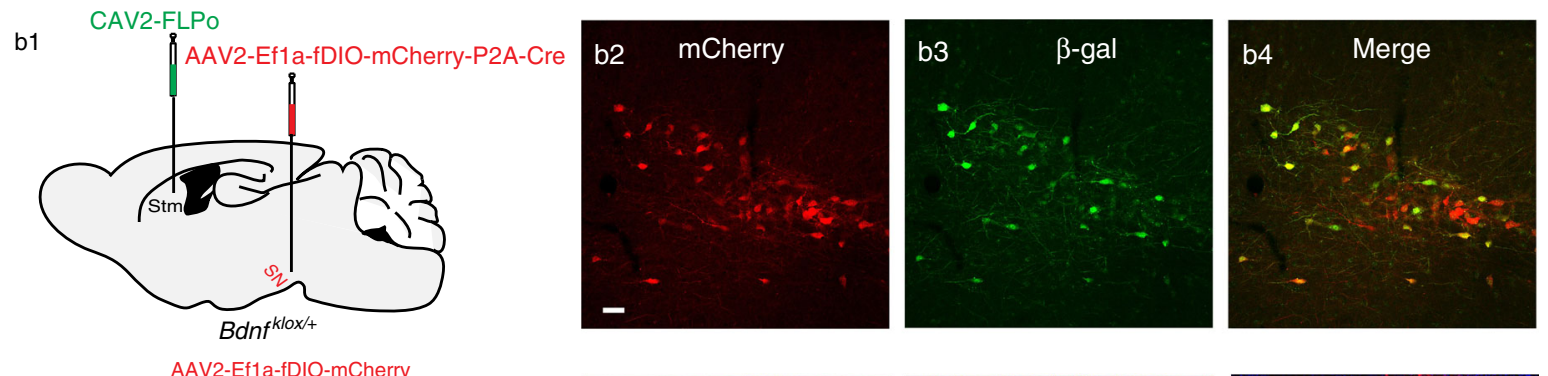

C $\mathrm{c} 1$

$$
\text { AAV2-Ef1a-fDIO-mCherry-P2A-Cre }
$$
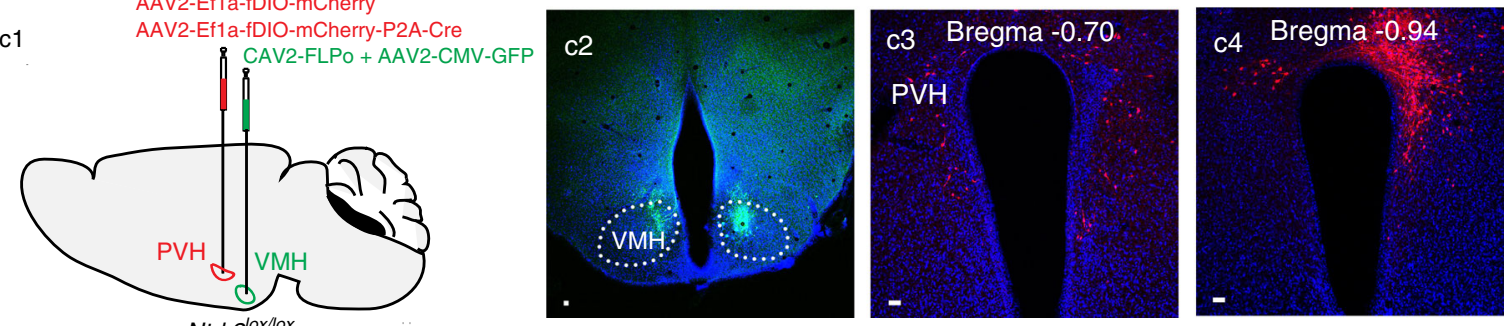

- AAV2-Ef1a-fDIO-mCherry - AAV2-Ef1a-fDIO-mCherry-P2A-Cre

d

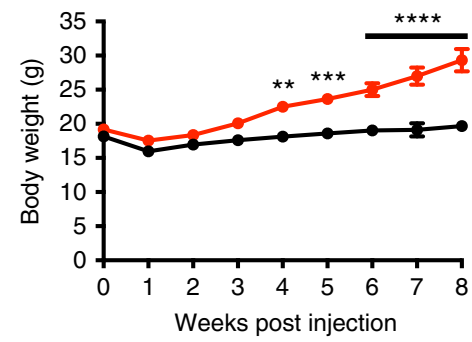

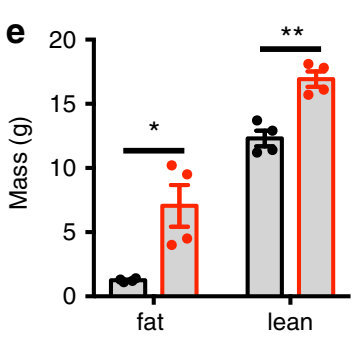

f

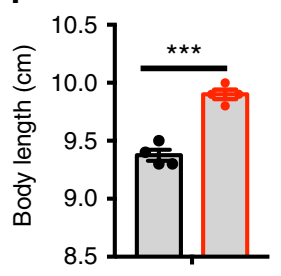

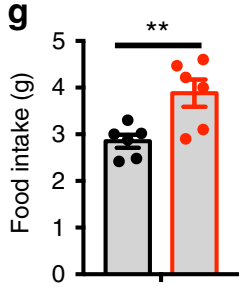

Fig. 6 Deletion of the Ntrk2 gene in PVH neurons projecting to the VMH leads to hyperphagia and obesity. a Diagrams of AAV2-Ef1a-fDIO-mCherry and AAV2-Ef1a-fDIO-mCherry-P2A-Cre viral vectors for FLP-dependent expression of mCherry and Cre. $\mathbf{b}$ Deletion of the Bdnf gene in substantia nigra neurons projecting to the striatum. CAV2-FLPo $(500 \mathrm{nl})$ and AAV2-Ef1a-fDIO-mCherry-P2A-Cre (200 nl) were stereotaxically injected into the striatum $(\mathrm{Stm})$ and substantia nigra (SN) of Bdnfklox/+ mice, respectively (b1). Expression of mCherry and $\beta$-galactosidase ( $\beta$-gal) was detected in the substantia nigra (b2-4). c Deletion of the Ntrk2 gene in PVH neurons projecting to the VMH. A mixture of CAV2-FLPo + AAV2-CMV-GFP (350 nl, 5:1 ratio) and either AAV2-Ef1a-fDIO-mCherry (control) or AAV2-Ef1a-fDIO-mCherry-P2A-Cre (150 nl) were bilaterally injected into the VMH and PVH of Ntrk2lox/lox mice, respectively (c1). Co-injected AAV2-CMV-GFP was used to mark CAV2 injection sites (c2). Neurons that are transduced by both CAV2-FLPo and one of the two FLP-dependent AAV vectors would express mCherry ( $c 3$ and $c 4)$. $\mathbf{d}$-g Body weight (d), fat mass and lean mass (e), body length (f), and daily food intake $(\mathbf{g})$ of female Ntrk2 ${ }^{l o x} /$ lox mice injected with CAV2-FLPo into the VMH and either AAV2-Ef1a-fDIO-mCherry or AAV2-Ef1a-fDIO-mCherryP2A-Cre into the PVH. $n=6$ mice for each group. Body weight data were analyzed using two-way ANOVA with Bonferroni post hoc tests; $F_{(1,90)}=124.0$, $P<0.0001$ for virus. Other data were analyzed with two-tailed unpaired $t$ test; ${ }^{\star} P=0.012$ and ${ }^{\star \star} P=0.002$ for fat mass and lean mass, ${ }^{\star \star \star} P=0.0002$ for body length, and ${ }^{\star \star \star} P=0.0097$ for food intake. Error bars indicate SEM. Scale bars represent $50 \mu \mathrm{m}$. Source data are provided as a Source Data file.

when CAV2-FLPo was not injected into the striatum (Supplementary Fig. 8a). We further confirmed no leaky expression of mCherry-P2A-Cre from AAV2-Ef1a-fDIO-mCherry-P2A-Cre by injecting the virus into the $\mathrm{PVH}$ (Supplementary Fig. 8b). This experiment shows that our two-virus system works for projection-specific gene deletion.

PVH TrkB regulates feeding through multiple projections. We targeted the Ntrk2 gene in PVH neurons that project to the NTS, VMH and LPBN, the three main projection fields of $\mathrm{PVH}^{\mathrm{TrkB}}$ neurons. Retrograde virus CAV2-FLPe-GFP (expressing a fusion protein of GFP and the improved FLP), CAV2-FLPo, or AAV2retro-hSyn-FLPo-T2A-GFP were used to express FLP in cell bodies of afferent neurons. We conducted this study only in female mice. Because Ntrk2 deletion in the PVH produced more severe obesity in females than males (Fig. 3b and Supplementary Fig. 4h), we used female mice to more sensitively uncover the role of different $\mathrm{PVH}^{\mathrm{TrkB}}$ projections in the control of appetite.

To delete the Ntrk2 gene in PVH neurons projecting to the NTS $\left(\mathrm{PVH}^{\mathrm{TrkB}} \rightarrow \mathrm{NTS}\right.$ neurons), we injected CAV2-FLPe-GFP into the NTS and either AAV2-Efla-fDIO-mCherry (control) or AAV2Efla-fDIO-mCherry-P2A-Cre into the PVH in Ntrk2lox/lox mice (Supplementary Fig. 8c). Because the NTS is a long structure, we infused a mixture of CAV2-FLPe-GFP and GRBs into two sites, the rostral part (Supplementary Fig. 8d1-3) and the caudal part (Supplementary Fig. 8d4-6) of the central NTS. Co-injected 
GRBs allows us to determine if an injection hits the NTS (Supplementary Fig. 8d). In hit mice, we detected mCherry expression in the PVH (Supplementary Fig. 8e), indicating expression of Cre in AAV2-Ef1a-fDIO-mCherry-P2A-Cre injected mice, which would delete the Ntrk2 gene in positive neurons. We found comparable body weight (Supplementary Fig. 8f), body composition (Supplementary Fig. 8g) and food intake (Supplementary Fig. 8h) between mutant and control mice. These results suggest that $\operatorname{TrkB}$ in $\mathrm{PVH}^{\mathrm{TrkB}} \rightarrow \mathrm{NTS}$ neurons is not essential for the control of food intake and body weight.

We next targeted the Ntrk2 gene in PVH neurons projecting to the $\mathrm{VMH}\left(\mathrm{PVH} \mathrm{TrkB}^{\mathrm{T}} \rightarrow \mathrm{VMH}\right.$ neurons) by injecting a mixture of CAV2-FLPo and AAV2-CMV-GFP into the VMH and either AAV2-Ef1a-fDIO-mCherry or AAV2-Ef1a-fDIO-mCherry-P2ACre into the PVH in Ntrk2lox/lox mice (Fig. 6c1). Co-injected AAV2-CMV-GFP marks injection sites (Fig. 6c2). In our initial trial, we noticed lesions at CAV2-FLPo injection sites, likely due to an immune response to CAV2. Therefore, we treated Ntrk2lox/lox mice with the immunosuppressant cyclophosphamide $(50 \mathrm{mg} / \mathrm{kg}$, i.p.) once every 2 days from day -3 to day 13 as previously described $^{58}$. Ten weeks after CAV2 injection, we did not detect any lesions in the VMH (Fig. 6c2), indicating that cyclophosphamide successfully blocked the immune response to CAV2. In mice with successful viral injection as evidenced by bilateral mCherry expression in the PVH (Fig. 6c3, c4), the Cre group had significantly higher body weight (Fig. 6d), fat mass and lean mass (Fig. 6c), body length (Fig. 6f), and food intake (Fig. 6g). These results show that deleting Ntrk2 in $\mathrm{PVH}^{\mathrm{TrkB}} \rightarrow \mathrm{VMH}$ neurons leads to hyperphagia and obesity, indicating a crucial role for $\operatorname{TrkB}$ in $\mathrm{PVH}^{\mathrm{TrkB}} \rightarrow \mathrm{VMH}$ neurons in appetite suppression.

Finally, we deleted the Ntrk2 gene in PVH neurons projecting

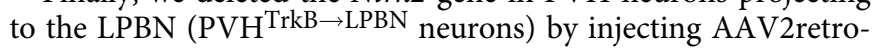
hSyn-FLPo-T2A-GFP into the LPBN and either AAV2-Ef1afDIO-mCherry or AAV2-Ef1a-fDIO-mCherry-P2A-Cre into the PVH in Ntrk2lox/lox mice (Fig. 7a1). We employed AAV2retrohSyn-FLPo-T2A-GFP rather than CAV2-FLPo for the PVH $\rightarrow$ LPBN projection, because we found that AAVretro is more efficient in retrograde infection than CAV2 at this projection. AAV2retro-hSyn-FLPo-T2A-GFP expresses GFP at injection sites (Fig. 7a2), allowing us to assess the accuracy of each injection. In mice with successful viral injection as evidenced by GFP expression in the LPBN (Fig. 7a2) and mCherry expression in the PVH (Fig. 7a3, a4), the Cre group had significantly higher body weight (Fig. 7b), fat mass and lean mass (Fig. 7c), body length (Fig. 7d), and food intake (Fig. 7e). These results show that deleting Ntrk2 in $\mathrm{PVH}^{\mathrm{TrkB}} \rightarrow \mathrm{LPBN}$ neurons also leads to hyperphagia and obesity, indicating a crucial role for TrkB in $\mathrm{PVH}^{\mathrm{TrkB}} \rightarrow$ LPBN neurons in the control of food intake.

VMH- and LPBN-projecting PVH TrkB neurons are distinct. Because the TrkB receptor at both $\mathrm{PVH}^{\mathrm{TrkB}}$ projections to the VMH and the LPBN is crucial for the control of appetite, we next investigated the possibility that the same subset of $\mathrm{PVH}^{\mathrm{TrkB}}$ neurons send collaterals to the VMH and the LPBN. We stereotaxically injected retrograde tracers CTB488 and Alexa Fluor 594-conjugated CTB (CTB594) into the VMH and the LPBN, respectively (Fig. 7f1-3). We found that CTB594 mainly labeled cell bodies in the rostral and medial parts of the PVH, whereas CTB488 largely in the medial and caudal parts of the PVH (Fig. 7f4-7). No neurons were detected to contain both CTB488 and CTB594 (Fig. 7f4-7). These results indicate that two distinct subtypes of PVH ${ }^{\text {TrkB }}$ neurons project to the VMH and the LPBN. We validated this finding by examining axonal terminals of mCherry-labeled PVH neurons that project to the LPBN in mice where AAV2-Ef1a-fDIO-mCherry and AAV2retro-hSyn-FLPo-
T2A-GFP were injected into the PVH and the LPBN, respectively (Supplementary Fig. 9a-d). We detected axonal terminals of these neurons in the LPBN, but not in the VMH (Supplementary Fig. $9 \mathrm{e}-\mathrm{h})$.

The observation that abolishment of Ntrk2 expression in $\mathrm{PVH}^{\mathrm{TrkB}} \rightarrow \mathrm{VMH}$ and $\mathrm{PVH}^{\mathrm{TrkB}} \rightarrow \mathrm{LPBN}$ neurons leads to hyperphagic obesity suggests that these two subtypes of neurons respond to changes in nutritional state. We tested this hypothesis by examining Fos induction in these two subsets of neurons in response to refeeding. We injected CTB488 into either the $\mathrm{VMH}$ (Fig. 8a) or the LPBN (Fig. 8d) of tamoxifen-treated Ntrk2 $2^{C r e E R /+}$; Rosa2 $6^{A i 9 /+}$ mice. After the mice were extensively handled, they were fasted overnight and a half of them were refed for $2 \mathrm{~h}$. We found that more CTB488-labeled $\mathrm{PVH}^{\mathrm{T}}{ }^{\mathrm{T} k B}$ neurons expressed Fos in refed mice than in fasted mice (Fig. $8 b, c, e, f$ ), indicating that refeeding activates $\mathrm{PVH}^{\mathrm{TrkB}} \rightarrow \mathrm{VMH}$ and $\mathrm{PVH}^{\mathrm{TrkB}} \rightarrow \mathrm{LPBN}$ neurons. These results together with the findings from Ntrk2 deletion and manipulation of neuronal activity, shows that feeding activates $\mathrm{PVH}^{\mathrm{TrkB} \rightarrow \mathrm{VMH}}$ and $\mathrm{PVH}^{\mathrm{TrkB}} \rightarrow \mathrm{LPBN}$ neurons to suppress appetite.

BDNF increases firing of $\mathrm{PVH}^{\mathrm{TrkB}}$ neurons. BDNF has been shown to potentiate neurotransmission at excitatory synapses ${ }^{19}$. As the vast majority of PVH neurons are excitatory ${ }^{18,19}$ and activation of $\mathrm{PVH}^{\mathrm{TrkB}}$ neurons suppress food intake (Fig. 4e, f), we tested the hypothesis that BDNF increases the activity of $\mathrm{PVH}^{\mathrm{TrkB}}$ neurons to suppress appetite. We injected AAV2-Ef1afDIO-mCherry and AAV2retro-Ef1a-DIO-FLPo into the PVH and the LPBN of $\mathrm{Ntrk2} 2^{\mathrm{CreER} /+}$ mice, respectively, to mark $\mathrm{PVH}^{\mathrm{TrkB}} \rightarrow \mathrm{LPBN}$ neurons with mCherry (Fig. 9a). Whole-cell patch-clamp recordings of these labeled neurons (Fig. 9b, c) revealed that bath application of $\operatorname{BDNF}(25 \mathrm{ng} / \mathrm{ml})$ increased neuronal firing with a latency of $15-40 \mathrm{~min}$ (Fig. 9d, e).

\section{Discussion}

This study has identified the $\mathrm{PVH}$ as a main site where TrkB signaling works to suppress food intake. Using a projectionspecific gene deletion strategy, we have further narrowed down the action site of TrkB signaling to two distinct subtypes of PVH neurons that project to the LPBN and the $\mathrm{VMH}$, respectively. We have found that refeeding activates these two subsets of $\mathrm{PVH}^{\mathrm{TrkB}}$ neurons, BDNF application increases the firing rate of $\mathrm{PVH}^{\mathrm{TrkB}} \rightarrow$ LPBN neurons, and chemogenetic activation of $\mathrm{PVH}^{\mathrm{TrkB}}$ neurons inhibits food intake during the dark cycle or after overnight food deprivation. Thus, our study indicates that signals reflecting repletion induce BDNF release, which in turn increases the activity of $\mathrm{PVH}^{\mathrm{TrkB}}$ neurons to promote satiety through their outputs to the LPBN and the VMH.

We found that deletion of the Ntrk2 gene with the Sim1-Cre transgene led to hyperphagia and reduced energy expenditure in mice. This observation is in agreement with the previous finding that BDNF expressed in the PVH suppresses food intake and promotes adaptive thermogenesis ${ }^{22}$. Collectively, these results demonstrate that BDNF-TrkB signaling plays a critical role in the control of both energy intake and energy expenditure. When we selectively deleted the Ntrk2 gene in the PVH using stereotaxic injection of Cre-expressing AAV, mutant mice displayed marked hyperphagia but normal energy expenditure. It is likely that TrkB signaling in other brain areas that express Sim1-Cre is involved in the regulation of energy expenditure. One of these brain areas could be the $\mathrm{DMH}$, in which Ntrk2 deletion significantly reduces energy expenditure ${ }^{28}$. Injection of BDNF protein into the $\mathrm{PVH}$ was reported to increase energy expenditure ${ }^{39}$. This could be a result of activation of the $\operatorname{TrkB}$ receptor at axonal terminals of 
a

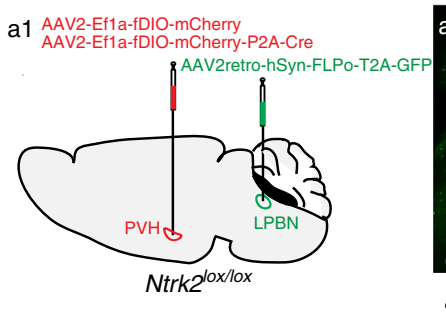

b

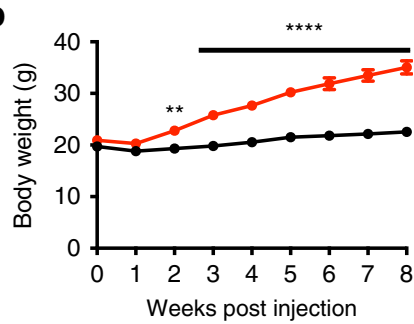

f

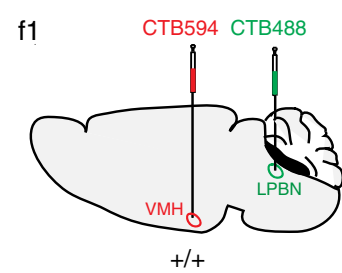

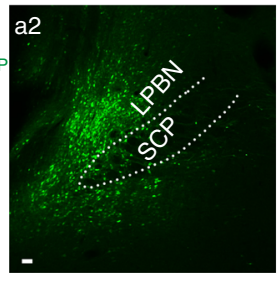

- AAV2-Ef1a-fDIO-mCherry

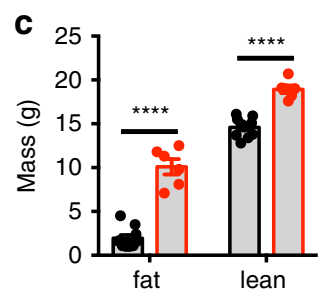

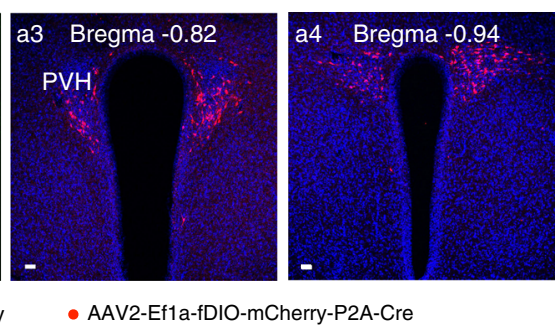

- AAV2-Ef1a-fDIO-mCherry-P2A-Cre

d
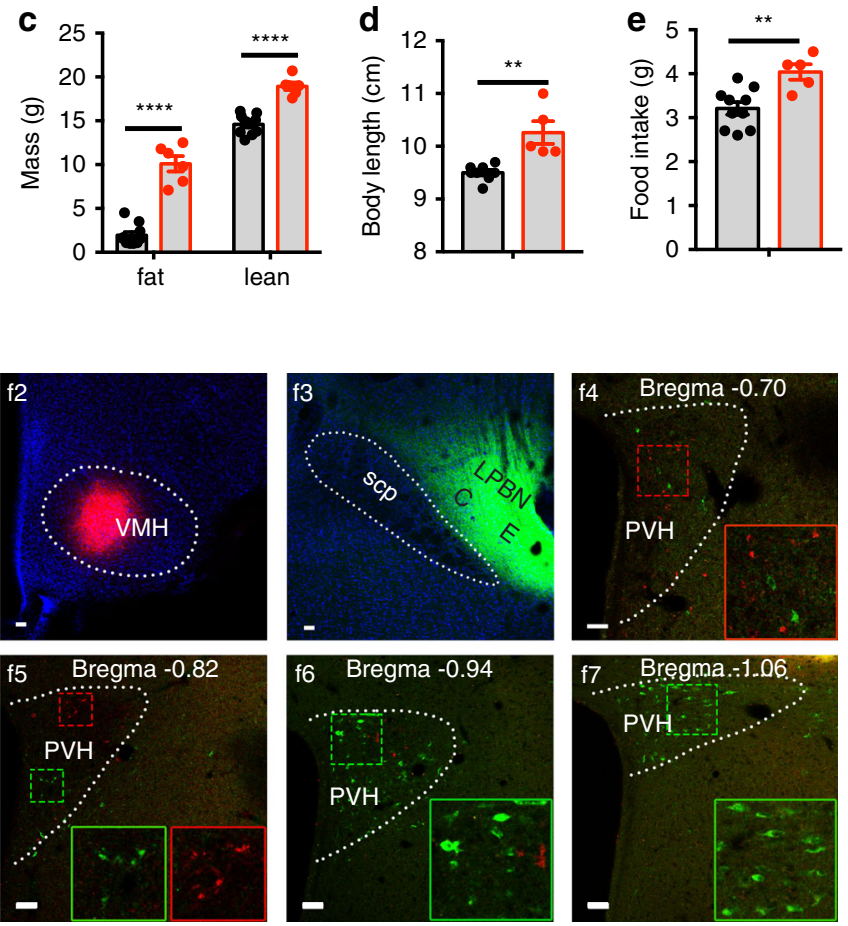

Fig. 7 Deletion of the Ntrk2 gene in PVH neurons projecting to the LPBN leads to hyperphagia and obesity. a Deletion of the Ntrk2 gene in PVH neurons projecting to the LPBN. AAV2retro-hSyn-FLPo-T2A-GFP (200 nl) and either AAV2-Ef1a-fDIO-mCherry (control) or AAV2-Ef1a-fDIO-mCherry-P2A-Cre $(150 \mathrm{nl})$ were bilaterally injected into the LPBN and PVH of Ntrk2lox/lox mice, respectively (a1). GFP marks injection sites of AAV2retro-hSyn-FLPo-T2A-GFP (a2). Neurons that are transduced by both AAV2retro-hSyn-FLPo-T2A-GFP and one of the two FLP-dependent AAV vectors would express mCherry (a3 and a4). b-e Body weight (b), fat mass and lean mass (c), body length (d), and food intake (e) of female Ntrk2lox/lox mice injected with AAV2retro-hSynFLPo-T2A-GFP into the LPBN and either AAV2-Ef1a-fDIO-mCherry or AAV2-Ef1a-fDIO-mCherry-P2A-Cre into the PVH. $n=10$ mice in the control group and 6 mice in the Cre group. Body weight data were analyzed using two-way ANOVA with Bonferroni post hoc tests; $F_{(1,126)}=488.7, P<0.0001$ for virus. Other data were analyzed with two-tailed unpaired $t$ test; ${ }^{\star \star \star \star} P<0.0001$ for fat mass and lean mass, ${ }^{\star \star} P=0.0014$ for body length, and ${ }^{\star \star} P=0.004$ for food intake. f Retrogradely labeling of PVH neurons. CTB594 (250 nl) and CTB488 (200 $\mathrm{nl}$ ) were injected into the VMH and the LPBN, respectively (f1-3). Some retrogradely labeled neurons are in the PVH (f4-7). Error bars indicate SEM. Scale bars represent $50 \mu \mathrm{m}$. Source data are provided as a Source Data file.

neurons that project to the PVH or a non-specific effect of recombinant BDNF.

A genetically defined group of neurons in a specific brain structure usually have multiple projection fields, and the powerful optogenetic approach is commonly employed to uncover the role of the neurons at each projection in animal behaviors. However, this approach is not suitable for uncovering gene function and is not ideal for examination of the long-term effect of altering neuronal activity. The projection-specific gene deletion described here makes it possible to determine the function of a gene in a specific neurocircuit. If deletion of the gene also alters the function of the targeted neurocircuit, this experiment will also reveal both short-term and long-term impacts of altering the activity of the circuit on behaviors and physiology.

Using the projection-specific gene deletion approach, we have identified two subtypes of $\mathrm{PVH}^{\text {TrkB }}$ neurons $\left(\mathrm{PVH}^{\mathrm{TrkB}} \rightarrow \mathrm{LPBN}\right.$ neurons and $\mathrm{PVH}^{\mathrm{TrkB}} \rightarrow \mathrm{VMH}$ neurons) in which TrkB signaling is necessary to suppress appetite. Deletion of the Ntrk2 gene in either of these two subtypes of neurons led to hyperphagia and obesity. Conversely, we found that deletion of the Ntrk2 gene in $\mathrm{PVH}{ }^{\mathrm{TrkB}} \rightarrow \mathrm{NTS}$ neurons did not affect energy balance. It is unlikely that this negative outcome is due to inadequate Ntrk2 deletion in NTS-projecting PVH neurons, as we showed that even unilateral Ntrk2 deletion in the PVH led to obesity. We have found that refeeding activates both $\mathrm{PVH}^{\mathrm{TrkB} \rightarrow \mathrm{LPBN}}$ and $\mathrm{PVH}^{\mathrm{TrkB}} \rightarrow \mathrm{VMH}$ subsets of neurons and that chemogenetic stimulation/inhibition of $\mathrm{PVH}^{\mathrm{TrkB}}$ neurons greatly decreases/ increases food intake, respectively. These results suggest that abolishment of TrkB signaling impairs the function of these two subtypes of neurons, which leads to deficits in transmitting satiation signals to their target neurons in the LPBN and VMH. These results also indicate that the $\mathrm{PVH}^{\mathrm{TrkB}} \rightarrow \mathrm{LPBN}$ and $\mathrm{PVH}^{\mathrm{TrkB}} \rightarrow \mathrm{VMH}$ neurocircuits are satiating and that BDNF is a key modulator of the two anorexigenic neurocircuits. As BDNF potently potentiates synaptic transmission ${ }^{19}$, it is likely that TrkB signaling enhances synaptic transmission in the two subtypes of 
a

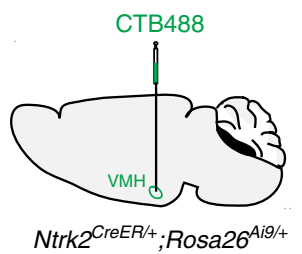

C

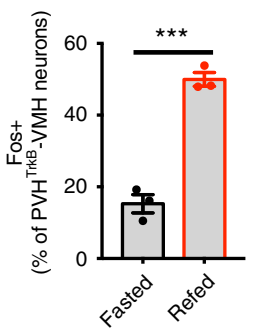

d

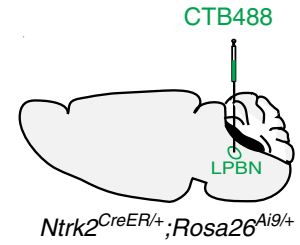

f

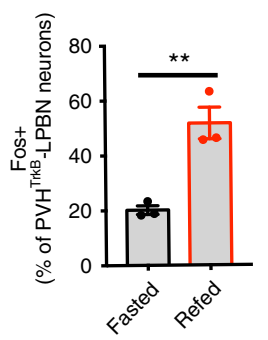

b
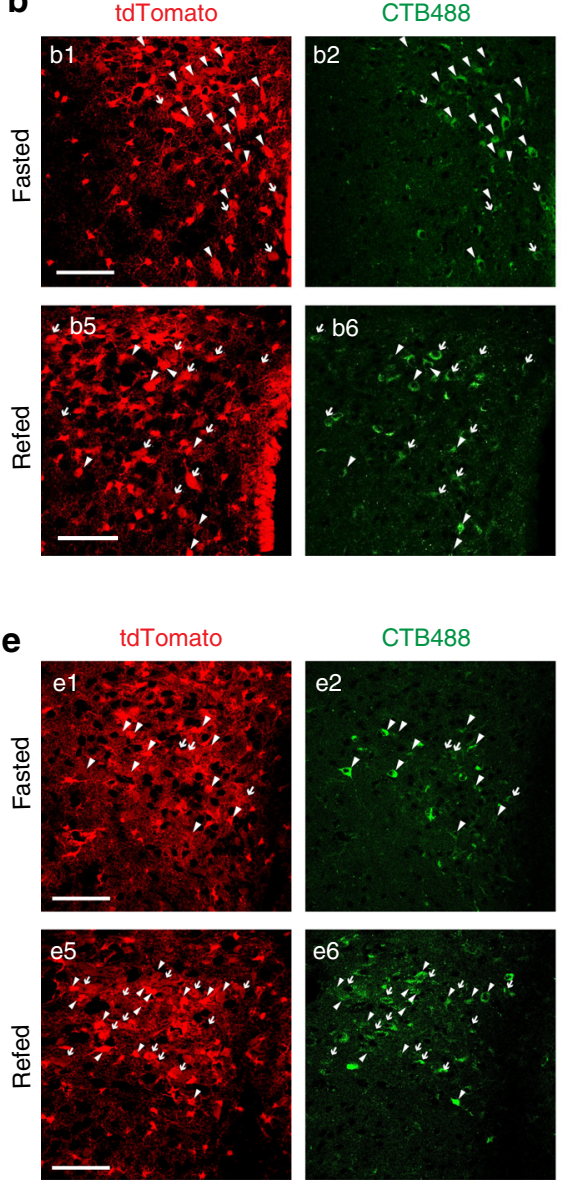
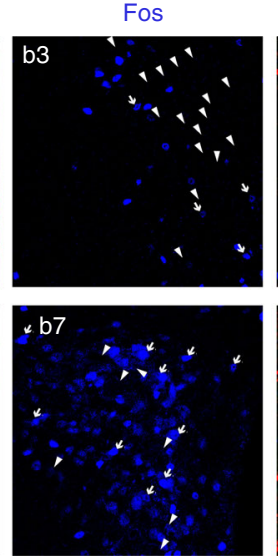

Fos
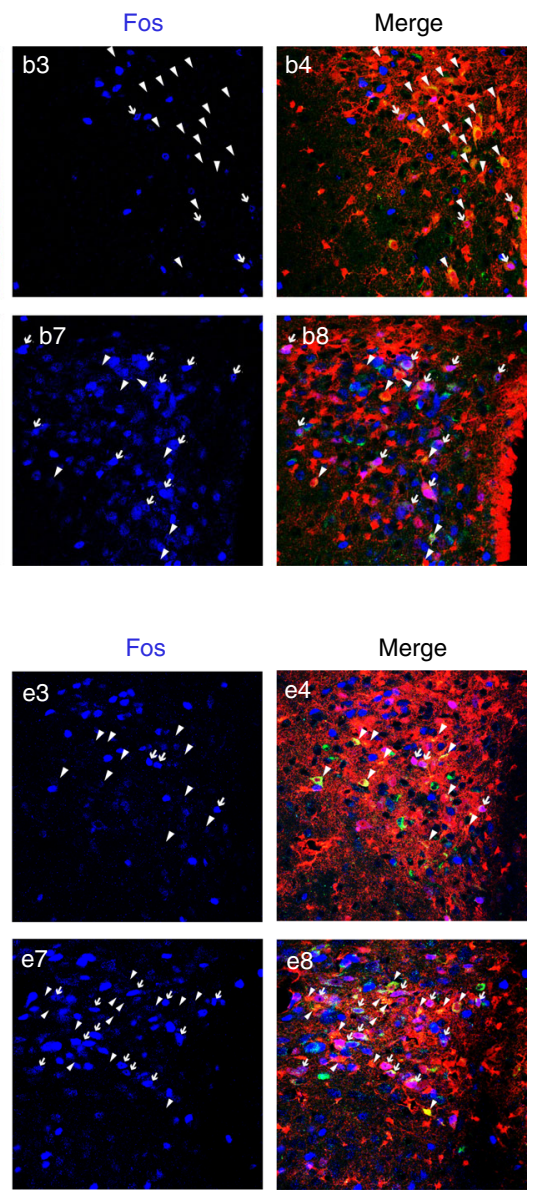

Fig. 8 Refeeding activates $\mathbf{P V H}^{\text {TrkB }}$ neurons projecting to the VMH and the LPBN. a-c Refeeding after overnight food deprivation induced Fos expression in $\mathrm{PVH}^{\mathrm{TrkB}}$ neurons projecting to the $\mathrm{VMH}$. PVH ${ }^{\mathrm{TrkB}} \rightarrow \mathrm{VMH}$ neurons were labeled by tdTomato and CTB488 injected into the $\mathrm{VMH}$ in tamoxifen-treated Ntrk2CreER/+;Rosa26Aig/+ mice (a). Arrowheads denote neurons positive for tdTomato and CTB488, while arrows indicate neurons positive for tdTomato, CTB488 and Fos (b). Fos induction in PVH TrkB $\rightarrow \mathrm{VMH}$ neurons under fasting and refeeding conditions was quantified (c). $\mathrm{n}=3$ mice in the fasted group and three mice in the refed group. Two-tailed unpaired $t$ test; ${ }^{* \star \star} P=0.0004$. $\mathbf{d}$-f Refeeding after overnight food deprivation induced Fos expression in PVH ${ }^{T r k B}$ neurons projecting to the LPBN. PVH ${ }^{T r k B} \rightarrow$ LPBN neurons were labeled by tdTomato and CTB488 injected into the LPBN in tamoxifen-treated Ntrk2CreER/+; Rosa26Ai9/+ mice (d). Arrowheads denote neurons positive for tdTomato and CTB488, while arrows indicate neurons positive for tdTomato, CTB488 and Fos (e). Fos induction in PVH ${ }^{T r k B} \rightarrow$ LPBN neurons under fasting and refeeding conditions was quantified (f). $n=3$ mice in the fasted group and three mice in the refed group. Two-tailed unpaired $t$ test; ${ }^{\star} P=0.006$. Error bars indicate SEM. Scale bars represent $50 \mu \mathrm{m}$. Source data are provided as a Source Data file.

PVH ${ }^{\text {TrkB }}$ neurons. Indeed, we found that BDNF application increased the firing rate of $\mathrm{PVH} \mathrm{TrkB}^{\mathrm{T}} \rightarrow \mathrm{LPBN}$ neurons. It would be important to understand which neurons release BDNF to facilitate activation of $\mathrm{PVH}^{\mathrm{TrkB}}$ neurons in response to refeeding, what are the targets of $\mathrm{PVH}^{\mathrm{TrkB}}$ neurons in the LPBN and VMH, and how TrkB signaling modulates synaptic function in $\mathrm{PVH}^{\mathrm{TrkB}} \rightarrow \mathrm{LPBN}$ and $\mathrm{PVH} \mathrm{T}^{\mathrm{TrkB}} \rightarrow \mathrm{VMH}$ neurons in future studies.

The vast majority of $\mathrm{PVH}$ neurons are glutamatergic neurons $^{59}$. These neurons have been shown to provide excitatory drive to the AgRP neurons in the arcuate nucleus ${ }^{60}$, the $\mathrm{CLPBN}^{26}$, and the pre-locus coeruleus ( $\mathrm{pLC})^{27}$ to regulate satiety. This study identifies one more neurocircuit out of the $\mathrm{PVH}$, i.e. $\mathrm{PVH}^{\mathrm{TrkB}} \rightarrow \mathrm{VMH}$, to regulate satiety.

Although ablation of the oxytocin receptor-expressing neurons in the hindbrain leads to overeating ${ }^{45}$, ablation of $\mathrm{PVH}$ oxytocin neurons in adult mice has no effect on body weight, food intake, or energy expenditure ${ }^{50}$. Consistent with this observation, we found that deletion of the Ntrk2 gene in $\mathrm{PVH}^{\mathrm{OXT}}$ neurons did not affect energy balance. Thus, $\mathrm{PVH}^{\mathrm{TrkB}} \rightarrow \mathrm{LPBN}$ and $\mathrm{PVH}^{\mathrm{TrkB}} \rightarrow \mathrm{VMH}$ neurons should be distinct from oxytocin neurons. Previous work shows that $\mathrm{LPBN}$-projecting $\mathrm{PVH}^{\mathrm{MC} 4 \mathrm{R}}\left(\mathrm{PVH}^{\mathrm{MC}} 4 \mathrm{R} \rightarrow \mathrm{LPBN}\right)$ neurons and $\mathrm{PVH}^{\mathrm{BDNF}}$ neurons also promote satiety ${ }^{22,26}$, although it remains unknown where $\mathrm{PVH}^{\mathrm{BDNF}}$ neurons project to suppress appetite. As we detected low co-localization of TrkB with either MC4R or BDNF, $\mathrm{PVH}^{\mathrm{TrkB}} \rightarrow \mathrm{LPBN}$ and $\mathrm{PVH}^{\mathrm{TrkB}} \rightarrow \mathrm{VMH}$ neurons could be distinct from $\mathrm{PVH}^{\mathrm{MC} 4 \mathrm{R} \rightarrow \mathrm{LPBN}}$ and $\mathrm{PVH}^{\mathrm{BDNF}}$ neurons. A recent study shows that $\mathrm{PDYN}$-expressing pLC-projecting $\mathrm{PVH}$ $\left(\mathrm{PVH}^{\mathrm{PDYN} \rightarrow \mathrm{pLC}}\right)$ neurons regulate satiety and are distinct from $\mathrm{PVH}^{\mathrm{MC} 4 \mathrm{R}}$ neurons ${ }^{27}$. If $\mathrm{PVH} \mathrm{BDNF}^{\mathrm{BDN}}$ neurons are distinct from $\mathrm{PVH}^{\mathrm{PDYN} \rightarrow \mathrm{pLC}}$ neurons, at least 5 subtypes of $\mathrm{PVH}$ neurons $\left(\mathrm{PVH}^{\mathrm{MC} 4 \mathrm{R} \rightarrow \mathrm{LPBN}}, \quad \mathrm{PVH}{ }^{\mathrm{BDNF}}, \quad \mathrm{PVH} \mathrm{HDYN}^{\mathrm{PDLC}}, \quad \mathrm{PVH}{ }^{\mathrm{TrkB}} \rightarrow \mathrm{LPBN}\right.$, and $\mathrm{PVH}^{\mathrm{TrkB}} \rightarrow \mathrm{VMH}$ ) have been identified to promote satiety. It would be important to investigate how these neurons coordinately regulate satiety and body weight. This is especially true for $\mathrm{PVH}^{\mathrm{MC}} 4 \mathrm{R} \rightarrow \mathrm{LPBN}$ and $\mathrm{PVH}^{\mathrm{TrkB}} \rightarrow \mathrm{LPBN}$ neurons, which project to the same target field, the cLPBN. It would be intriguing to know whether these two subtypes of neurons innervate the same neurons in the CLPBN and receive inputs from the same afferent neurons. 
a

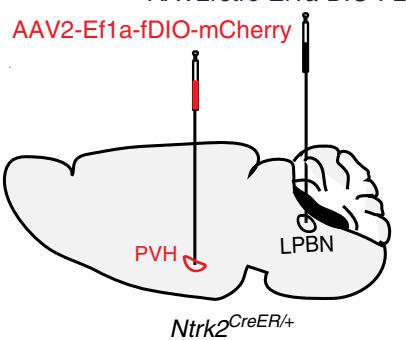

d

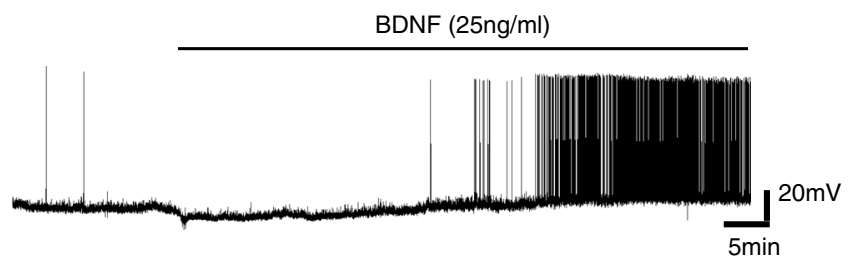

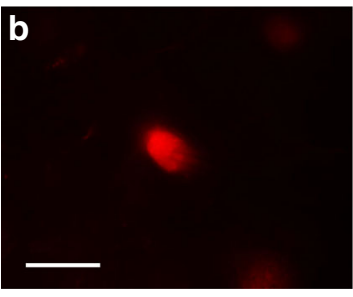
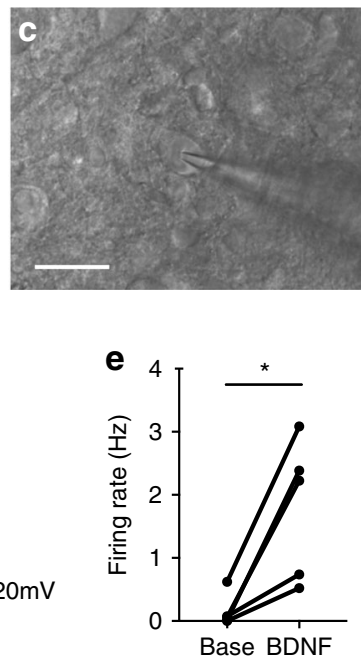

Fig. 9 BDNF increases the firing rate of PVH TrkB neurons projecting to the LPBN. a $P V H^{T r k B} \rightarrow L P B N$ neurons were labeled with injection of $A A V 2-E f 1 a-$ fDIO-mCherry into the PVH and AAV2retro-Ef1a-DIO-FLPo into the LPBN of Ntrk2CreER/+ mice. $\mathbf{b}$, $\mathbf{c}$ Patch-clamp recording of an mCherry-labeled $P V H^{T r k B} \rightarrow L P B N$ neuron. Scale bars represent $25 \mu \mathrm{m}$. d A representative trace showing neuronal firing before and after bath application of BDNF. e Quantification of baseline neuronal firing (Base) and neuronal firing 40 min after bath application of BDNF (BDNF). Two-tailed paired Student's $t$ test; ${ }^{\star} P=0.019$. Source data are provided as a Source Data file.

These future studies will provide insights into the organization and function of the neural network that regulates appetite.

\section{Methods}

Animals. Floxed Ntrk2 (Ntrk2 $2^{\text {lox }}$; also known as TrkB $\left.{ }^{l o x}\right)^{43}$, floxed Ntrk2-LacZ $\left(N \operatorname{trk} 2^{f B Z} \text {; also known as } \mathrm{fBZ}\right)^{42}$, and floxed $B d n f\left(B d n f^{k l o x}\right)^{56}$ mouse strains were previously described. Ntrk2 $2^{l o x}$ mice were backcrossed to C57BL/6J mice for at least 15 generations before they were used in this study. Sim1-Cre (stock No: 006395), MC4Rtau-GFP (stock No: 008323), Rosa26 ${ }^{A i 9}$ [Gt9(ROSA)26Sor tm99CAG-tdTomato)Hze/ J; stock No: 007909] and C57BL6/J (stock No: 000664) mouse strains were obtained from the Jackson Laboratory. The Ntrk2 ${ }^{\mathrm{CreER} /+}$ (also known as TrkB CreER) mouse strain $^{32}$ was kindly provided by Dr. David Ginty at Harvard Medical School. We generated $\mathrm{Ntrk2} 2^{\mathrm{CreER} /+} ; \mathrm{Rosa}_{2} 6^{\mathrm{Aig} /+}$ mice by crossing $\mathrm{Ntrk2} 2^{\mathrm{CreER} /+}$ mice to Rosa $26^{A i 9 /+}$ mice. To induce nuclear localization of the CreER fusion protein in mice harboring the Ntrk2 ${ }^{C r e E R}$ allele, we treated the mice with tamoxifen $(2 \mathrm{mg}$ per animal, i.p.) once a day for 7 consecutive days in 6-8-week-old mice. Animals were used for experiments 7 days after the last tamoxifen injection. Ntrk $2^{\text {lox } / l o x}$ mice were crossed with Sim1-Cre;Ntrk2lox/lox mice to generate Sim1-Cre;Ntrk2 $2^{\text {lox } / l o x}$ mutant mice and Ntrk2lox/lox control mice. All mice used in this study were maintained in the C56BL6/J genetic background. Mice were maintained on a 12-h/ 12-h light/dark cycle with ad libitum access to water and regular laboratory chow (Teklad Rodent Diet 2019; $3.3 \mathrm{kcal} / \mathrm{g}$ energy density, 22\% kcal from fat) unless otherwise specified. All experiments were performed in accordance with relevant guidelines and regulations regarding the use of experimental animals. The Animal Care and Use Committees at The Scripps Research Institute Florida approved all animal procedures used in this study.

Immunohistochemistry. For neuropeptide immunohistochemistry, we injected Ntrk $2^{C r e E R /+}$;Rosa $26^{A i 9 /+}$ mice with colchicine $(20 \mu \mathrm{g}$ per animal, i.c.v.; Tocris $)$ to impair protein transportation in neuronal processes. Forty-eight hours after colchicine injection, mice were deeply anaesthetized with avertin and transcardially perfused with phosphate buffered saline (PBS), followed by $4 \%$ paraformaldehyde in PBS. Coronal brain sections $(40 \mu \mathrm{m}$ in thickness) were obtained using a sliding microtome (Leica SM2000R). Brain sections were rinsed once with Tris buffered saline (TBS; $10 \mathrm{mM}$ Tris- $\mathrm{HCl}, 150 \mathrm{mM} \mathrm{NaCl}, \mathrm{pH} 7.5$ ), incubated with blocking buffer $(0.4 \%$ Triton $\mathrm{X}-100,1 \%$ bovine serum albumin, and $10 \%$ goat serum or horse serum in TBS) for $1 \mathrm{~h}$ at room temperature, and then incubated with primary antibodies diluted in the blocking buffer overnight at room temperature. The following primary antibodies were used: chicken anti- $\beta$-galactosidase $(1: 3,000$; abcam \#ab9361), rabbit anti-NeuN (1:1000; Millipore \#ABN78), rabbit anti-GFAP (1:400; Sigma \#G9269), mouse anti-oxytocin (1:5000; Millipore \#MAB5296), rabbit anti-CRH (1:500; Millipore \#AB1760), mouse anti-GFP (1:1000; Clontech \#632460), rabbit anti-somatostatin (1:500; immunostar \#20067), rabbit anti-GHRH (1:500; Immunostar \#22938), rabbit anti-vasopressin (1:5000; Millipore \#AB1565), mouse anti-tyrosine hydroxylase (1:10,000; Sigma \#T1299), rabbit anti-c-Fos antibody (1:5000; abcam \#ab208942), rabbit anti-prodynorphin (1:200; abcam \#ab11137), rabbit anti-DsRed (1:1000; TaKaRa \#632496), and rabbit anti-TRH (1:10,000; generous gift from Dr. Martin Wessendorf, University of Minnesota). After three washes in TBS, sections were incubated with appropriate fluorescent secondary antibodies (1;500; Jackson ImmunoResearch) for $1 \mathrm{~h}$ at room temperature. Sections were washed three times in TBS, mounted onto slides, and coverslipped with a mounting medium containing 4',6-diamidino-2-phenylindole (DAPI; Vector Laboratories). Fluorescent images were captured using a Nikon $\mathrm{C} 2+$ confocal microscope or a Leica TCS SP8 microscope.

Plasmids and viruses. For generation of pAAV-Efla-fDIO-mCherry and pAAV Efla-fDIO-mCherry-P2A-Cre, pAAV-Efla-fDIO-EYFP (a gift from Karl Deisseroth; Addgene plasmid \#55641) was used as backbone. pAAV-Efla-fDIO-EYFP was digested with AscI and NheI site to remove EYFP. The sequence encoding mCherry and mCherry-P2A-Cre were obtained by PCR using plasmid pLM-CMVR-Cre (a gift from Michel Sadelain; Addgene plasmid \#27546) as template. PCR products were digested with AscI and NheI and cloned into the backbone of pAAV-Efla-fDIO-EYFP to generate plasmid pAAV-Efla-fDIO-mCherry and pAAV-Ef1a-fDIO-mCherry-P2A-Cre. pAAV-hSyn-FLPo-T2A-GFP was generated using pAAV-hSynapsin-Flpo (a gift from Massimo Scanziani; Addgene plasmid \#60663) as backbone. We replaced the FLPo sequence containing a stop codon with a FLPo without a stop codon using BamHI and HindIII sites to generate an intermediate plasmid. The T2A-GFP sequence was amplified by PCR using plasmid PX461 (a gift from Feng Zhang; Addgene plasmid \#48140) as template and inserted into the intermediate plasmid at HindIII site to generate pAAV-hSynFLPo-T2A-GFP. AAV2-Ef1a-fDIO-mCherry, AAV2-Ef1a-fDIO-mCherry-P2ACre and AAV2retro-hSyn-Flpo-T2A-GFP were packaged by UNC Vector Core. Plasmid AAV pEF1a-DIO-FLPo-WPRE-hGHpA was obtained from Addgene (\#87306; a gift of Li Zhang), and AAV2retro-DIO-FLPo virus was packaged by ViGene Biosciences Inc. AAV2-CMV-GFP, AAV2-CMV-GFP-Cre and AAV2CAG-FLEX-tdTomato viruses were purchased from UNC vector Core. AAV2hSyn-DIO-mCherry, AAV2-hSyn-DIO-hM3Dq-mCherry and AAV2-hSyn-DIOhM4Di-mCherry were purchased from Addgene.

To construct pCAV2-FLPe-GFP, we inserted the Flpe-GFP sequence into the CAV shuttle plasmid ${ }^{61}$ to generate pShuttle-FLPe-GFP. The FLPe-GFP sequence was isolated from pCAG-Flpe-GFP (a gift from Connie Cepko, Addgene plasmid $\# 13788$ ). The AscI-PacI fragment of the shuttle construct was purified and used to generate pCAV2-FLPe-GFP through homologous recombination in E. coli as described $^{62}$. To generate pCAV2-FLPo, we began with pCAV-FLEx ${ }^{l o x}$-Flp ${ }^{63}$

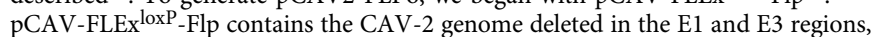
and a cassette containing a CMV promoter, two loxP sites, an inverted FLPo open reading frame, and an SV40 polyA signal. pCAV-FLEx ${ }^{\text {loxP }}$-Flp was incubated with recombinant Cre recombinase (New England Biolabs) and then transformed into $\mathrm{DH} \alpha$ bacteria. A clone containing the FLPo cassette in the $5^{\prime} \rightarrow 3^{\prime}$ orientation was identified by restriction digestion analyses and denoted pCAV2-FLPo. Viral vectors CAV2-FLPe-GFP and CAV2-FLPo were generated, amplified, and purified as previously described ${ }^{61}$. 
Stereotaxic injection of virus and CTB. Mice were deeply anesthetized with isoflurane and were placed onto a stereotaxic apparatus (David Kopf, Model 940). A small incision was made to expose the skull, and a small hole was drilled on skull above the injection site. A Nanofil 33-gauge needle (World Precision Instruments, \#NF33BV-2) was inserted into the brain and the virus was injected at a rate of $25 \mathrm{nl}$ per min using Microsyringe Pump (World Precision Instruments, Micro4). After infusion, the needle was stayed in the same position for $5 \mathrm{~min}$. Following injections, mice received subcutaneous injection of Loxicom $(0.5 \mathrm{mg}$ per $\mathrm{kg})$ to relieve pain. Stereotaxic coordinates from the bregma and skull used for this study were PVH (AP: $-0.5 \mathrm{~mm}, \mathrm{ML}: \pm 0.25 \mathrm{~mm}, \mathrm{DV}:-5.4 \mathrm{~mm}$ ), VMH (AP: $-1.4 \mathrm{~mm}, \mathrm{ML}: \pm$ $0.4 \mathrm{~mm}$, DV: $-5.95 \mathrm{~mm}$ ), LPBN (AP: $-5 \mathrm{~mm}$, ML: $\pm 1.5 \mathrm{~mm}, \mathrm{DV}:-3.8 \mathrm{~mm}$ ), vlPAG (AP: $-4.72 \mathrm{~mm}, \mathrm{ML}: \pm 0.68 \mathrm{~mm}, \mathrm{DV}:-2.70 \mathrm{~mm}$ ), and LC (AP: $-5.4 \mathrm{~mm}$, ML: $\pm 0.91 \mathrm{~mm}, \mathrm{DV}:-4.1 \mathrm{~mm}$ ). Stereotaxic coordinates for the NTS relative to the calamus scriptorius were rostral NTS (AP: $+0.85 \mathrm{~mm}, \mathrm{ML}: \pm 0.58 \mathrm{~mm}, \mathrm{DV}$ : $-0.42 \mathrm{~mm}$ ) and caudal NTS (AP: $-0.0 \mathrm{~mm}, \mathrm{ML}: \pm 0.26 \mathrm{~mm}$, DV: $-0.4 \mathrm{~mm}$ ). After physiological measurements are completed, animals were perfused with saline and $4 \%$ paraformaldehyde and their brains were sliced for examination of injection sites. Mice with missed injections were either assigned to a separate group or excluded in final data analyses. Cholera toxin b subunit conjugated with Alexa dye 488 (CTB-488; Life Technologies, \# C-34775) or 594 (CTB-594; Life Technologies, \# C-34777) at $1 \mathrm{mg}$ per $\mathrm{ml}$ was used for retrograde tracing.

\section{Physiological measurements. In the Ntrk2 gene deletion experiments, body} weight was measured weekly. Body length (naso-anal) was measured from anesthetized mice in the end of each experiment. For food intake, mice were individually housed, and daily food intake was measured over 5 days after 3 days of acclimation. Body composition was determined using a Minispec LF-50/mq 7.5 NMR Analyzer (Brucker Optics). $\mathrm{VO}_{2}$ and locomotor activity were assessed with a comprehensive lab animal monitoring system (CLAMS, Columbus Instruments). Ambulatory activity was measured as light beam breaks in the XY horizontal plane.

Chemogenetic manipulation of food intake. Mice injected with AAV2-hSynDIO-mCherry, AAV2-hSyn-DIO-hM3D(Gq)-mCherry or AAV2-hSyn-DIOhM4D(Gi)-mCherry were singly housed for at least 2 weeks with daily handling prior to experiment. For food intake measurement during light cycle, mice were transferred to a new cage without food at 7:30 am and injected intraperitoneally with vehicle or CNO (Sigma-Aldrich \#C08325) at 8:30 a.m. Pre-weighed food was then added to each cage at 9:00 a.m. and food intake was measured hourly for $3 \mathrm{~h}$. For food intake measurement during dark cycle, mice were transferred to a new cage without food at 3 p.m. and injected with vehicle or $\mathrm{CNO}$ at 5:30 pm. Preweighed food was then added to each cage at 6:00 p.m. and food intake was measured hourly from 7:00 p.m. to 9:00 p.m. For food intake measurement after overnight fasting, mice were transferred to a new cage with alpha-dri bedding without food at 6:00 p.m. for overnight fasting. On the following day, mice were injected with vehicle or $\mathrm{CNO}$ at 9:30 a.m. and pre-weighed food was added to each cage at 10:00 a.m. and food intake was measured hourly for $3 \mathrm{~h}$. The CNO dosage was $1 \mathrm{mg}$ per kg body weight for mice injected with AAV2-hSyn-DIO-hM3D(Gq)mCherry and $3 \mathrm{mg}$ per kg body weight for mice injected with AAV2-hSyn-DIOhM4D(Gi)-mCherry.

In situ hybridization. Freshly dissected mouse brains were quickly frozen in a 2methylbutane (Fisher Scientific \#O3551-4) dry ice bath. Subsequently, 20- $\mu$ m thick cryostat sections were collected on Superfrost Plus slides (Fisherbrand $\# 12-550-15)$ and used for in situ hybridization. Radioactive in situ hybridization of brain sections was performed using ${ }^{35} \mathrm{~S}$-labeled riboprobes as previously described ${ }^{11}$. To generate antisense riboprobes, the mouse cDNA sequence for Ntrk2 (GenBank accession number X17647, nucleotides 188-722) was amplified by PCR and cloned into pBluscript II KS (-) plasmid (Stratagene). Antisense probes were synthesized with T3 RNA polymerase (Promega \#2083) and labeled with alpha${ }^{35}$ S-CTP (PerkinElmer \#NEG064H250UC) and alpha- ${ }^{35}$ S-UTP (PerkinElmer \#NEG039H250UC). Brain sections were postfixed in $4 \%$ paraformaldehyde for $1 \mathrm{~h}$ and acetylated for $10 \mathrm{~min}$ with $0.1 \mathrm{M}$ triethanolamine hydrochloride $/ 0.25 \%$ acetic anhydride ( $\mathrm{pH} 8.0$ ). After dehydration, hybridization was performed with ${ }^{35} \mathrm{~S}$ labeled probes at $55^{\circ} \mathrm{C}$ for overnight. After hybridization, sections were treated with RNase A $(200 \mu \mathrm{g} / \mathrm{ml}$, Fisher Scientific \# BP2539100) to remove unannealed probes. After dehydration, sections were exposed to Kodak BioMax MR films (Carestream Health) for 3 days. Developed film was scanned at 1200 dpi for image processing. Fluorescence in situ hybridization was performed using the RNAscope Multiplex Fluorescent Reagent Kit v2 (\#323100, Advanced Cell diagnostics). Brain sections were fixed with pre-chilled $10 \%$ formalin at $4{ }^{\circ} \mathrm{C}$ for $15 \mathrm{~min}$, and dehydrated with $50 \%, 75 \%$, and $100 \%$ ethanol. Sections were air-dried for $20 \mathrm{~min}$, pretreated with protease IV for $30 \mathrm{~min}$, washed 2 times in PBS and incubated with the target probes (Ntrk2-C3; \#423611-C3, tdTomato-C2; \#317041-C2, Glp1r-C2; $\# 418851$ ) for $2 \mathrm{~h}$ at $40^{\circ} \mathrm{C}$. The signal was amplified by subsequent incubation with v2Amp1 for $30 \mathrm{~min}$, v2Amp2 for $30 \mathrm{~min}$, v2Amp3 for $15 \mathrm{~min}$, and followed by TSA Plus Fluorescein/Cyanine5 (NEL754001KT, Akoya Biosciences) for $30 \mathrm{~min}$ at $40{ }^{\circ} \mathrm{C}$. Slides were counterstained with DAPI and images were acquired using a Nikon $\mathrm{C} 2+$ confocal microscope.
Quantification of co-expression. For fluorescent in situ hybridization, brains were sectioned at $14-\mu \mathrm{m}$ thickness, and ten series of PVH-containing sections were collected (one slide for each series and 4-5 sections on each slide). One slide was used for each in situ hybridization. Sections on each slide approximately covers the PVH from Bregma -0.58 to $-1.06 \mathrm{~mm}$. In situ hybridization signals were examined for co-expression on each cell marked with DAPI staining in the PVH. For fluorescent immunohistochemistry, every other brain section $(40-\mu \mathrm{m}$ thick) from each $\mathrm{Ntrk2}{ }^{\mathrm{CreER} /+}$;Rosa26 $6^{\mathrm{Aig} /+}$ brain (6-7 sections in total) was used. Immunoreactive PVH neurons in all these sections were examined for co-expression with tdTomato. DAPI staining was used to determine the PVH structure. TrkB colocalization with oxytocin was examined in brain sections from two mice, while TrkB colocalization with other PVH markers was examined in brain sections from one mouse.

Quantitative real-time PCR. Mouse brain was rapidly extracted and placed in icecold 1x HBSS. Brain slice from bregma -0.34 to bregma -1.22 was obtained using a vibratome (Leica VT 1200s, Germany), and the PVH was punched out bilaterally using a 20 gauge blunt-end needle. The punched brain tissues were transferred from needle into $1.5-\mathrm{ml}$ tubes. Total RNA was isolated from PVH using PureLink RNA mini kit combined with Trizol reagent (Life Technologies, Carlsbad, CA). To remove genomic DNA contamination, the RNA samples were treated with RNasefree DNase. First strand cDNA was reverse transcribed using $50 \mathrm{ng}$ of total RNA and Super-Script ${ }^{\text {stx }}$ III (Invitrogen, Carlsbad, CA) according to the manufacture's protocol. Quantitative PCR was performed on step-one thermal cycler (Applied Biosystems, Foster City, CA) with SYBR ${ }^{\circledast}$ Green PCR master mix (Roche). Levels for Ntrk2 mRNA encoding the full-length TrkB (forward, gtgctgatggcagagggta; reverse, tattttcaccagcaggttctct), $M c 4 r$ mRNA (forward, cccggacggaggatgctat; reverse, tcgccacgatcactagaatgt),) were normalized over 18s rRNA (forward, ccgcagctggaataatgga; reverse, ccctcttaatcatggcctca) mRNA in the same sample. As the $M c 4 r$ gene does not contain an intron, a parallel experiment using no reverse transcriptase control was used to monitor genomic DNA contamination.

Slice whole-cell electrophysiology. Male mice at 12-16 weeks of were decapitated under isoflurane anesthesia. Brains were rapidly removed and placed in ice-cold sucrose cutting solution containing $(\mathrm{mM}): 72$ sucrose, $83 \mathrm{NaCl}, 2.5 \mathrm{KCl}, 1 \mathrm{CaCl}_{2}$ $5 \mathrm{MgCl}_{2}, 1 \mathrm{NaH}_{2} \mathrm{PO}_{4}, 22 \mathrm{D}$-glucose, and $26.0 \mathrm{NaHCO}_{3}$ saturated with $95 \% \mathrm{O}_{2} / 5 \%$ $\mathrm{CO}_{2}$. Coronal brain slices containing the $\mathrm{PVH}$ were obtained using a vibratome (Leica VT 1200s, Germany), and then transferred to artificial cerebrospinal fluid (aCSF) composed of (mM): $126 \mathrm{NaCl}, 2.5 \mathrm{KCl}, 21.4 \mathrm{NaHCO}_{3}, 1.2 \mathrm{NaH}_{2} \mathrm{PO}_{4}$, $1.2 \mathrm{MgCl}, 2.4 \mathrm{CaCl}_{2}$ and $10 \mathrm{D}$-glucose, equilibrated with $95 \% \mathrm{O}_{2}$ and $5 \% \mathrm{CO}_{2}$ at $32^{\circ} \mathrm{C}$ for $30 \mathrm{~min}$. Brain slices were maintained and recorded at room temperature $\left(22-25^{\circ} \mathrm{C}\right)$. Then, the brain slices were gently transferred to a recording chamber (RC-27, Warner Instruments, Hamden, CT), and the chamber was perfused with circulated oxygenated aCSF at a flow rate of $2-3 \mathrm{ml}$ per min. PVH TrkB neurons projecting to LPBN were visually identified in slices using an infrared-differential interference contrast microscope and Texas Red fluorescent filter (Scientifica, UK). Whole-cell patch-clamp recordings were performed using borosilicate glass pipettes (ID: $0.68 \mathrm{~mm}$, OD: $1.2 \mathrm{~mm}$, WPI, Sarasota, FL) of 3-5 M $\Omega$ pulled with a micropipette puller (P-1000; Sutter Instrument, Novato, CA). The intracellular solution for current clamp recording contained $(\mathrm{mM})$ : $128 \mathrm{~K}$-gluconate, $10 \mathrm{KCl}$, 10 HEPES, 1 EGTA, $1 \mathrm{MgCl}_{2}$, $5 \mathrm{Na}_{2}$-ATP, $0.3 \mathrm{Mg}$-GTP, $0.3 \mathrm{CaCl}_{2}$, adjusted to $\mathrm{pH}$ 7.3 with $\mathrm{KOH}$. To test the effect of BDNF on the activity of $\mathrm{PVH}^{\text {TrkB }}$ neurons projecting to LPBN, current clamp recordings were performed in continuous mode with membrane potential held between -55 and $-60 \mathrm{mV}$ to minimize the spontaneous firing rate ${ }^{64}$. Baseline activities were recorded at least for $20 \mathrm{~min}$, then BDNF (Millipore Corp, Billerica, MA) was added to the circulating aCSF to reach a final concentration of $25 \mathrm{ng} / \mathrm{ml}$. Signals were acquired with Multiclamp700B and Digidata 1550A (Molecular Devices, San Jose, CA). Data were low-pass filtered at $2.9 \mathrm{KHz}$ and sampled at $10 \mathrm{kHz}$. The neuronal firing rate was analyzed with Clampfit 10.6 software. Data were expressed as average firing rate within $20 \mathrm{~min}$ just before and 40 min post BDNF treatment.

Statistical analysis. Statistical analyses were performed using Prism 7 (GraphPad Software). All data are expressed as mean \pm SEM. Data were tested using two-tailed unpaired or paired $t$ test or two-way ANOVA with post hoc Bonferroni's or Tukey's multiple comparisons for statistical significance. Experiments in this study were repeated independently for two to three times. No statistical methods were used to determine sample size for each experiment. The animal numbers were estimated based on our previous studies. Half of the brain sections from each virusinjected mouse were used for histological examination of injection sites. Mice with weak or off-target viral expression were excluded from final data analysis. Each micrograph shows a representative situation for each group of mice.

Reporting summary. Further information on research design is available in the Nature Research Reporting Summary linked to this article. 


\section{Data availability}

The data that support the findings of this study are available from the corresponding author upon reasonable request. The source data underlying Figs. 1-9 and

Supplementary Figs. 1, 3-5, and 8 are provided as a Source Data file.

Received: 28 May 2019; Accepted: 12 March 2020;

Published online: 07 April 2020

\section{References}

1. Montague, C. T. et al. Congenital leptin deficiency is associated with severe early-onset obesity in humans. Nature 387, 903-908 (1997).

2. Yeo, G. S. et al. A frameshift mutation in MC4R associated with dominantly inherited human obesity. Nat. Genet 20, 111-112 (1998).

3. Vaisse, C., Clement, K., Guy-Grand, B. \& Froguel, P. A frameshift mutation in human MC4R is associated with a dominant form of obesity. Nat. Genet $\mathbf{2 0}$, 113-114 (1998).

4. Yeo, G. S. et al. A de novo mutation affecting human TrkB associated with severe obesity and developmental delay. Nat. Neurosci. 7, 1187-1189 (2004).

5. Han, J. C. et al. Brain-derived neurotrophic factor and obesity in the WAGR syndrome. N. Engl. J. Med. 359, 918-927 (2008).

6. Zhang, Y. et al. Positional cloning of the mouse obese gene and its human homologue. Nature 372, 425-432 (1994).

7. Tartaglia, L. A. et al. Identification and expression cloning of a leptin receptor, OB-R. Cell 83, 1263-1271 (1995).

8. Huszar, D. et al. Targeted disruption of the melanocortin-4 receptor results in obesity in mice. Cell 88, 131-141 (1997).

9. Yaswen, L., Diehl, N., Brennan, M. B. \& Hochgeschwender, U. Obesity in the mouse model of pro-opiomelanocortin deficiency responds to peripheral melanocortin. Nat. Med. 5, 1066-1070 (1999).

10. Rios, M. et al. Conditional deletion of brain-derived neurotrophic factor in the postnatal brain leads to obesity and hyperactivity. Mol. Endocrinol. 15, 1748-1757 (2001).

11. $\mathrm{Xu}, \mathrm{B}$. et al. Brain-derived neurotrophic factor regulates energy balance downstream of melanocortin-4 receptor. Nat. Neurosci. 6, 736-742 (2003).

12. Thorleifsson, G. et al. Genome-wide association yields new sequence variants at seven loci that associate with measures of obesity. Nat. Genet 41, 18-24 (2009).

13. Speliotes, E. K. et al. Association analyses of 249,796 individuals reveal 18 new loci associated with body mass index. Nat. Genet. 42, 937-948 (2010).

14. Okada, Y. et al. Common variants at CDKAL1 and KLF9 are associated with body mass index in east Asian populations. Nat. Genet. 44, 302-306 (2012).

15. Wen, W. et al. Meta-analysis identifies common variants associated with body mass index in east Asians. Nat. Genet. 44, 307-311 (2012).

16. Andermann, M. L. \& Lowell, B. B. Toward a wiring diagram understanding of appetite control. Neuron 95, 757-778 (2017).

17. Huang, E. J. \& Reichardt, L. F. Neurotrophins: roles in neuronal development and function. Annu Rev. Neurosci. 24, 677-736 (2001).

18. Waterhouse, E. G. \& Xu, B. New insights into the role of brain-derived neurotrophic factor in synaptic plasticity. Mol. Cell Neurosci. 42, 81-89 (2009).

19. Park, H. \& Poo, M. M. Neurotrophin regulation of neural circuit development and function. Nat. Rev. Neurosci. 14, 7-23 (2013).

20. Lee, K. F. et al. Targeted mutation of the gene encoding the low affinity NGF receptor p75 leads to deficits in the peripheral sensory nervous system. Cell 69, 737-749 (1992).

21. Baeza-Raja, B. et al. p75 Neurotrophin receptor regulates energy balance in obesity. Cell Rep. 14, 255-268 (2016).

22. An, J. J., Liao, G. Y., Kinney, C. E., Sahibzada, N. \& Xu, B. Discrete BDNF neurons in the paraventricular hypothalamus control feeding and energy expenditure. Cell Metab. 22, 175-188 (2015).

23. Unger, T. J., Calderon, G. A., Bradley, L. C., Sena-Esteves, M. \& Rios, M. Selective deletion of Bdnf in the ventromedial and dorsomedial hypothalamus of adult mice results in hyperphagic behavior and obesity. J. Neurosci. 27, 14265-14274 (2007).

24. Yang, H., An, J. J., Sun, C. \& Xu, B. Regulation of energy balance via bdnf expressed in nonparaventricular hypothalamic neurons. Mol. Endocrinol. 30, 494-503 (2016).

25. Liao, G. Y., Kinney, C. E., An, J. J. \& Xu, B. TrkB-expressing neurons in the dorsomedial hypothalamus are necessary and sufficient to suppress homeostatic feeding. Proc. Natl Acad. Sci. USA 116, 3256-3261 (2019).

26. Garfield, A. S. et al. A neural basis for melanocortin-4 receptor-regulated appetite. Nat. Neurosci. 18, 863-871 (2015).

27. Li M. M. et al. The paraventricular hypothalamus regulates satiety and prevents obesity via two genetically distinct circuits. Neuron. 102, 653-667 (2019).
28. Li, C. et al. Defined Paraventricular hypothalamic populations exhibit differential responses to food contingent on caloric state. Cell Metab. 29, 681-694 e685 (2019).

29. Swanson, L. W. \& Sawchenko, P. E. Hypothalamic integration: organization of the paraventricular and supraoptic nuclei. Annu Rev. Neurosci. 6, 269-324 (1983).

30. Biag, J. et al. Cyto- and chemoarchitecture of the hypothalamic paraventricular nucleus in the C57BL/6J male mouse: a study of immunostaining and multiple fluorescent tract tracing. J. Comp. Neurol. 520, 6-33 (2012).

31. Simmons, D. M. \& Swanson, L. W. Comparison of the spatial distribution of seven types of neuroendocrine neurons in the rat paraventricular nucleus: toward a global 3D model. J. Comp. Neurol. 516, 423-441 (2009).

32. Rutlin, M. et al. The cellular and molecular basis of direction selectivity of Adelta-LTMRs. Cell 159, 1640-1651 (2014).

33. Madisen, L. et al. A robust and high-throughput Cre reporting and characterization system for the whole mouse brain. Nat. Neurosci. 13, 133-140 (2010).

34. Fenner, B. M. Truncated TrkB: beyond a dominant negative receptor. Cytokine growth factor Rev. 23, 15-24 (2012).

35. Liu, H. et al. Transgenic mice expressing green fluorescent protein under the control of the melanocortin-4 receptor promoter. J. Neurosci. 23, 7143-7154 (2003).

36. Li, M. M. et al. The paraventricular hypothalamus regulates satiety and prevents obesity via two genetically distinct circuits. Neuron 102, 653-667 e656 (2019).

37. Liao, G. Y., Li, Y. \& Xu, B. Ablation of TrkB expression in RGS9-2 cells leads to hyperphagic obesity. Mol. Metab. 2, 491-497 (2013).

38. Wang, C., Bomberg, E., Billington, C., Levine, A. \& Kotz, C. M. Brain-derived neurotrophic factor in the hypothalamic paraventricular nucleus reduces energy intake. Am. J. Physiol. Regul. Integr. Comp. Physiol. 293, R1003-R1012 (2007).

39. Wang, C., Bomberg, E., Billington, C., Levine, A. \& Kotz, C. M. Brain-derived neurotrophic factor in the hypothalamic paraventricular nucleus increases energy expenditure by elevating metabolic rate. Am. J. Physiol. Regul. Integr. Comp. Physiol. 293, R992-R1002 (2007).

40. Balthasar, N. et al. Divergence of melanocortin pathways in the control of food intake and energy expenditure. Cell 123, 493-505 (2005).

41. $\mathrm{Xu}, \mathrm{B}$. et al. Cortical degeneration in the absence of neurotrophin signaling: dendritic retraction and neuronal loss after removal of the receptor TrkB. Neuron 26, 233-245 (2000).

42. $\mathrm{Xu}, \mathrm{B}$. et al. The role of brain-derived neurotrophic factor receptors in the mature hippocampus: modulation of long-term potentiation through a presynaptic mechanism involving TrkB. J. Neurosci. 20, 6888-6897 (2000)

43. Baydyuk, M. et al. TrkB receptor controls striatal formation by regulating the number of newborn striatal neurons. Proc. Natl Acad. Sci. USA 108, 1669-1674 (2011)

44. Bariohay, B. et al. Brain-derived neurotrophic factor/tropomyosin-related kinase receptor type B signaling is a downstream effector of the brainstem melanocortin system in food intake control. Endocrinology 150, 2646-2653 (2009).

45. Baskin, D. G. et al. A new oxytocin-saporin cytotoxin for lesioning oxytocinreceptive neurons in the rat hindbrain. Endocrinology 151, 4207-4213 (2010).

46. Kublaoui, B. M., Gemelli, T., Tolson, K. P., Wang, Y. \& Zinn, A. R. Oxytocin deficiency mediates hyperphagic obesity of Siml haploinsufficient mice. Mol. Endocrinol. 22, 1723-1734 (2008).

47. Swaab, D. F., Purba, J. S. \& Hofman, M. A. Alterations in the hypothalamic paraventricular nucleus and its oxytocin neurons (putative satiety cells) in Prader-Willi syndrome: a study of five cases. J. Clin. Endocrinol. Metab. 80, 573-579 (1995).

48. Holder, J. L. Jr, Butte, N. F. \& Zinn, A. R. Profound obesity associated with a balanced translocation that disrupts the SIM1 gene. Hum. Mol. Genet 9, 101-108 (2000).

49. Traurig, M. et al. Common variation in SIM1 is reproducibly associated with BMI in Pima Indians. Diabetes 58, 1682-1689 (2009)

50. $\mathrm{Wu}, \mathrm{Z}$. et al. An obligate role of oxytocin neurons in diet induced energy expenditure. PLoS ONE 7, e45167 (2012).

51. Alexander, G. M. et al. Remote control of neuronal activity in transgenic mice expressing evolved G protein-coupled receptors. Neuron 63, 27-39 (2009).

52. Krashes, M. J. et al. Rapid, reversible activation of AgRP neurons drives feeding behavior in mice. J. Clin. Invest 121, 1424-1428 (2011).

53. Campos, C. A., Bowen, A. J., Roman, C. W. \& Palmiter, R. D. Encoding of danger by parabrachial CGRP neurons. Nature 555, 617-622 (2018)

54. Soudais, C., Laplace-Builhe, C., Kissa, K. \& Kremer, E. J. Preferential transduction of neurons by canine adenovirus vectors and their efficient retrograde transport in vivo. FASEB J. 15, 2283-2285 (2001).

55. Tervo, D. G. et al. A designer AAV variant permits efficient retrograde access to projection neurons. Neuron 92, 372-382 (2016). 
56. Liao, G. Y. et al. Dendritically targeted Bdnf mRNA is essential for energy balance and response to leptin. Nat. Med 18, 564-571 (2012).

57. Baydyuk, M., Xie, Y., Tessarollo, L. \& Xu, B. Midbrain-derived neurotrophins support survival of immature striatal projection neurons. J. Neurosci. 33, 3363-3369 (2013).

58. Ariza, L. et al. Central nervous system delivery of helper-dependent canine adenovirus corrects neuropathology and behavior in mucopolysaccharidosis type VII mice. Hum. Gene Ther. 25, 199-211 (2014).

59. Vong, L. et al. Leptin action on GABAergic neurons prevents obesity and reduces inhibitory tone to POMC neurons. Neuron 71, 142-154 (2011)

60. Krashes, M. J. et al. An excitatory paraventricular nucleus to AgRP neuron circuit that drives hunger. Nature 507, 238-242 (2014).

61. Kremer, E. J., Boutin, S., Chillon, M. \& Danos, O. Canine adenovirus vectors: an alternative for adenovirus-mediated gene transfer. J. Virol. 74, 505-512 (2000).

62. Szelechowski M., Bergeron C., Gonzalez-Dunia D., Klonjkowski B. Production and purification of non replicative canine adenovirus type 2 derived vectors. $J$. Vis. Exp. 82, e50833 (2013).

63. Schwarz, L. A. et al. Viral-genetic tracing of the input-output organization of a central noradrenaline circuit. Nature 524, 88-92 (2015).

64. Ghamari-Langroudi, M. et al. G-protein-independent coupling of MC4R to Kir7.1 in hypothalamic neurons. Nature 520, 94-98 (2015).

\section{Acknowledgements}

We thank David Ginty for the Ntrk2 ${ }^{C r e E R}$ mouse strain, Martin Wessendorf for the antiTRH antibody, and Jessica Houtz and Shaw-wen Wu for critical reading of this paper. This work was supported by US National Institutes of Health grants to BX (R01 DK103335 and R01 DK105954).

\section{Author contributions}

J.J.A. and B.X. conceived the study. J.J.A., C.E.K., and B.X. designed the study. J.J.A. did colocalization immunohistochemistry and analyzed Sim1-Cre;Ntrk2lox/lox conditional knockout mice. C.E.K., J.J.A., and G.Y.L. did stereotaxic injection of AAV-Cre-GFP. C.E. K. and J.J.A. analyzed PVH-specific Ntrk2 mutant mice. J.J.A. constructed viral vectors for projection-specific gene deletion, did DREADD experiments, and examined Fos induction after refeeding. J.J.A. constructed pCAV2-FLPe-GFP. E.J.K. produced CAV2-FLPe-GFP and CAV2-FLPo. J.J.A. and C.E.K. did projection tracing and projection-specific Ntrk2 deletion. J.W.T performed electrophysiological recordings. J.J. A. and B.X. prepared figures. B.X. and J.J.A. wrote the paper with input from all authors.

\section{Competing interests}

The authors declare no competing interests.

\section{Additional information}

Supplementary information is available for this paper at https://doi.org/10.1038/s41467020-15537-w.

Correspondence and requests for materials should be addressed to B.X.

Peer review information Nature Communications thanks Maribel Rios, Zhiping Pang and the other anonymous reviewer(s) for their contribution to the peer review of this work. Peer reviewer reports are available.

Reprints and permission information is available at http://www.nature.com/reprints

Publisher's note Springer Nature remains neutral with regard to jurisdictional claims in published maps and institutional affiliations.

cC Open Access This article is licensed under a Creative Commons Attribution 4.0 International License, which permits use, sharing, adaptation, distribution and reproduction in any medium or format, as long as you give appropriate credit to the original author(s) and the source, provide a link to the Creative Commons license, and indicate if changes were made. The images or other third party material in this article are included in the article's Creative Commons license, unless indicated otherwise in a credit line to the material. If material is not included in the article's Creative Commons license and your intended use is not permitted by statutory regulation or exceeds the permitted use, you will need to obtain permission directly from the copyright holder. To view a copy of this license, visit http://creativecommons.org/ licenses/by/4.0/

(C) The Author(s) 2020 